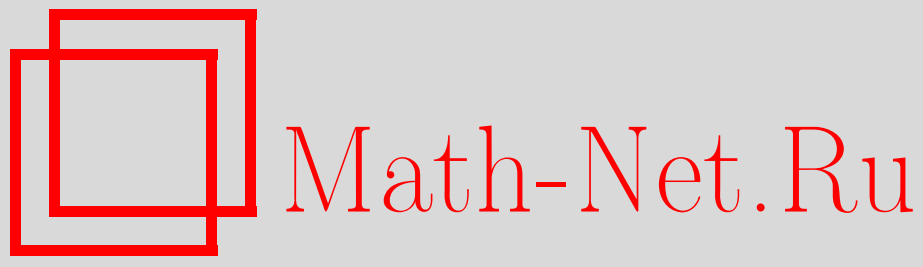

М. Ш. Бирман, Т. А. Суслина, Усреднение стационарной периодической системы Максвелла в случае постоянной магнитной проницаемости, Функи. анализ и его прил., 2007, том 41, выпуск 2, 3-23

DOI: https://doi.org/10.4213/faa2859

Использование Общероссийского математического портала MathNet.Ru подразумевает, что вы прочитали и согласны с пользовательским соглашением

http://www . mathnet.ru/rus/agreement

Параметры загрузки:

IP : 54.80 .97 .219

26 апреля 2023 г., $17: 27: 51$

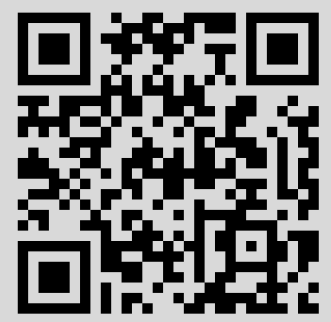


Функииональный анализ и его приложения

2007, т. 41, вып. 2, с. 3-23

УДК 517.956

\title{
Усреднение стационарной периодической системы Максвелла в случае постоянной магнитной проницаемости*
}

\author{
(с) 2007. М. Ш. Бирман, Т. А. СУСЛинА
}

Посвящается светлой памяти великого математика Марка Григоръевича Крейна

\section{Введение}

0.1. Постановка задачи. В работе изучается задача об усреднении (гомогенизации) стационарной периодической системы Максвелла в $\mathbb{R}^{3}$. Эта задача много изучалась традиционными методами теории усреднений (см., например, $[2],[9],[1],[8])$. Теоретико-операторный подход, предложенный авторами в статьях [3], [4], применялся к системе Максвелла в [4, гл. 7] и в [10], [11]. Начнем с обзора соответствующих результатов.

Ниже через $L_{2}\left(\mathbb{R}^{3} ; \mathbb{C}^{n}\right)$ обозначается $L_{2}$-пространство $\mathbb{C}^{n}$-значных функций в $\mathbb{R}^{3}$, а $H^{s}\left(\mathbb{R}^{3} ; \mathbb{C}^{n}\right), s>0,-$ соответствующие классы Соболева. При $n=1$ пишем просто $L_{2}\left(\mathbb{R}^{3}\right), H^{s}\left(\mathbb{R}^{3}\right)$. Положим

$$
\mathfrak{G}=L_{2}\left(\mathbb{R}^{3} ; \mathbb{C}^{3}\right), \quad J=\{\mathbf{f} \in \mathfrak{G}: \operatorname{div} \mathbf{f}=0\} .
$$

Пусть $\Gamma$ - решетка периодов в $\mathbb{R}^{3}$ и $\Omega$ - элементарная ячейка решетки $Г$. Диэлектрическая проницаемость $\eta(\mathbf{x})$ и магнитная проницаемость $\mu(\mathbf{x})$ суть $Г$-периодические матрицы, ограниченные и положительно определенные. Через $\mathbf{u}$, $\mathbf{v}$ обозначаются напряженности электрического и магнитного полей, $\mathbf{w}=\eta \mathbf{u}-$ индукция электрического поля, $\mathbf{z}=\mu \mathbf{v}$ - индукция магнитного поля. Оператор Максвелла $\mathscr{M}$ мы записываем в терминах индукций, считая векторы w и z соленоидальными. Тогда оператор $\mathscr{M}=\mathscr{M}(\eta, \mu)$ действует в пространстве $J \oplus J$ и задается формулой

$$
\mathscr{M}(\eta, \mu)=\left(\begin{array}{cc}
0 & i \operatorname{rot} \mu^{-1} \\
-i \operatorname{rot} \eta^{-1} & 0
\end{array}\right)
$$

на естественной области определения (см. ниже формулу (2.1)). Точка $\lambda=i$ является регулярной точкой для оператора $\mathscr{M}$.

Пусть $\varepsilon>0$ - параметр. Рассмотрим семейство операторов $\mathscr{M}_{\varepsilon}=\mathscr{M}\left(\eta^{\varepsilon}, \mu^{\varepsilon}\right)$ с быстро осциллирующими при $\varepsilon \rightarrow 0$ коэффициентами $\eta^{\varepsilon}(\mathbf{x})=\eta\left(\varepsilon^{-1} \mathbf{x}\right), \mu^{\varepsilon}(\mathbf{x})$ $=\mu\left(\varepsilon^{-1} \mathbf{x}\right)$. Мы изучаем поведение резольвенты $\left(\mathscr{M}_{\varepsilon}-i I\right)^{-1}$ при $\varepsilon \rightarrow 0$. Иначе говоря, речь идет о поведении при $\varepsilon \rightarrow 0$ решений $\mathbf{w}_{\varepsilon}, \mathbf{z}_{\varepsilon}$ уравнения

$$
\left(\mathscr{M}_{\varepsilon}-i I\right)\left(\begin{array}{c}
\mathbf{w}_{\varepsilon} \\
\mathbf{z}_{\varepsilon}
\end{array}\right)=\left(\begin{array}{l}
\mathbf{q} \\
\mathbf{r}
\end{array}\right), \quad \mathbf{q}, \mathbf{r} \in J,
$$

\footnotetext{
*Работа выполнена при поддержке РФФИ (гранты 05-01-01076-a, 05-01-02944-ЯФ-а).
} 
а также соответствующих напряженностей $\mathbf{u}_{\varepsilon}=\left(\eta^{\varepsilon}\right)^{-1} \mathbf{w}_{\varepsilon}, \mathbf{v}_{\varepsilon}=\left(\mu^{\varepsilon}\right)^{-1} \mathbf{z}_{\varepsilon}$. В подробной записи уравнение (0.1) имеет вид

$$
\left.\begin{array}{r}
\operatorname{rot}\left(\mu^{\varepsilon}\right)^{-1} \mathbf{z}_{\varepsilon}-\mathbf{w}_{\varepsilon}=-i \mathbf{q}, \\
\operatorname{rot}\left(\eta^{\varepsilon}\right)^{-1} \mathbf{w}_{\varepsilon}+\mathbf{z}_{\varepsilon}=i \mathbf{r}, \quad \operatorname{div} \mathbf{z}_{\varepsilon}=0 .
\end{array}\right\}
$$

«Усредненный» (эффективный) оператор Максвелла $\mathscr{M}^{0}=\mathscr{M}\left(\eta^{0}, \mu^{0}\right)$ определяется коэффициентами $\eta^{0}, \mu^{0}$, где $\eta^{0}$ (соответственно $\left.\mu^{0}\right)$ - эффективная матрица, отвечающая эллиптическому оператору $-\operatorname{div} \eta(\mathbf{x}) \nabla$ (соответственно $-\operatorname{div} \mu(\mathbf{x}) \nabla)$. (Определение матрицы $\eta^{0}$ приведено ниже, см. (1.4), (1.5).) «Эллиптическое» правило усреднения коэффициентов для системы Максвелла хорошо известно. Пусть $\mathbf{w}_{0}, \mathbf{z}_{0}$ - решение «усредненной» системы

$$
\left(\mathscr{M}^{0}-i I\right)\left(\begin{array}{l}
\mathbf{w}_{0} \\
\mathbf{z}_{0}
\end{array}\right)=\left(\begin{array}{l}
\mathbf{q} \\
\mathbf{r}
\end{array}\right)
$$

и $\mathbf{u}_{0}=\left(\eta^{0}\right)^{-1} \mathbf{w}_{0}, \mathbf{v}_{0}=\left(\mu^{0}\right)^{-1} \mathbf{z}_{0}$. Известно (см., например, [2], [9], [1], [8]), что вектор-функции $\mathbf{u}_{\varepsilon}, \mathbf{v}_{\varepsilon}, \mathbf{w}_{\varepsilon}, \mathbf{z}_{\varepsilon}$ слабо сходятся в $\mathfrak{G}$ к полям $\mathbf{u}_{0}, \mathbf{v}_{0}, \mathbf{w}_{0}, \mathbf{z}_{0}$ соответственно. Близкие результаты известны и для нестационарной системы Максвелла.

0.2. Применение подхода, предложенного в [3], [4], позволяет взамен слабой сходимости получать равномерные аппроксимащии по норме в $\mathfrak{G}$ с точной по порядку оценкой остатка. До сих пор, однако, удавалось получить такие аппроксимации не для всех полей. Поясним это подробнее.

Полезно представить каждое из полей в виде суммы двух слагаемых: $\mathbf{w}_{\varepsilon}=$ $\mathbf{w}_{\varepsilon}^{(q)}+\mathbf{w}_{\varepsilon}^{(r)}, \mathbf{z}_{\varepsilon}=\mathbf{z}_{\varepsilon}^{(q)}+\mathbf{z}_{\varepsilon}^{(r)}$, где векторы $\mathbf{w}_{\varepsilon}^{(q)}, \mathbf{z}_{\varepsilon}^{(q)}$ являются решением системы (0.2) при $\mathbf{r}=0$, а векторы $\mathbf{w}_{\varepsilon}^{(r)}, \mathbf{z}_{\varepsilon}^{(r)}$ - решением системы $(0.2)$ при $\mathbf{q}=0$. Соответственно в виде сумм представляются и поля $\mathbf{u}_{\varepsilon}, \mathbf{v}_{\varepsilon}$. Таким образом, фактически мы интересуемся поведением восъми полей при $\varepsilon \rightarrow 0$.

В гл. 7 работы [4] рассматривался случай $\mu=\mathbf{1}$. (Впрочем, рассуждения из [4, гл. 7] автоматически обобщаются и на случай постоянного $\mu$.$) При этом$ $\mathbf{v}_{\varepsilon}=\mathbf{z}_{\varepsilon}$, и речь идет о шести полях. Для одного из них, а именно для магнитной напряженности $\mathbf{v}_{\varepsilon}^{(r)}=\mathbf{z}_{\varepsilon}^{(r)}$, была получена аппроксимация по норме в $\mathfrak{G}$ :

$$
\left\|\mathbf{v}_{\varepsilon}^{(r)}-\mathbf{v}_{0}^{(r)}\right\|_{\mathfrak{G}} \leqslant C \varepsilon\|\mathbf{r}\|_{\mathfrak{G},}, \quad 0<\varepsilon \leqslant 1
$$

Здесь поле $\mathbf{v}_{0}^{(r)}=\mathbf{z}_{0}^{(r)}$ является (вместе с $\mathbf{w}_{0}^{(r)}$ ) решением усредненной системы (0.3) при $\mathbf{q}=0$ (и $\left.\mu^{0}=\mathbf{1}\right)$.

Метод из [4, гл. 7] состоит в сведении к уравнению второго порядка. Поле $\mathbf{z}_{\varepsilon}^{(r)}$ (см. систему $(0.2)$ при $\mathbf{q}=0, \mu^{\varepsilon}=\mathbf{1}$ ) является решением задачи

$$
\operatorname{rot}\left(\eta^{\varepsilon}\right)^{-1} \operatorname{rot} \mathbf{z}_{\varepsilon}^{(r)}+\mathbf{z}_{\varepsilon}^{(r)}=i \mathbf{r}, \quad \operatorname{div} \mathbf{z}_{\varepsilon}^{(r)}=0 .
$$

Затем система (0.5) расширяется, и дело сводится к изучению эллиптического оператора второго порядка

$$
\mathscr{L}_{\varepsilon}=\mathscr{L}\left(\eta^{\varepsilon}, \mathbf{1}, 1\right)=\operatorname{rot}\left(\eta^{\varepsilon}\right)^{-1} \operatorname{rot}-\nabla \operatorname{div},
$$


действующего в пространстве $\mathfrak{G}$ (ср. с оператором (1.1) ниже). Этот оператор входит в общий класс ДО (дифференциальных операторов), выделенный и изученный в [3], [4]. К нему применим общий результат из [3], [4] об аппроксимации резольвенты по операторной норме в $\mathfrak{G}$ через резольвенту эффективного опеpaтора $\mathscr{L}^{0}$ :

$$
\left\|\left(\mathscr{L}_{\varepsilon}+I\right)^{-1}-\left(\mathscr{L}^{0}+I\right)^{-1}\right\|_{\mathfrak{G} \rightarrow \mathfrak{G}} \leqslant C \varepsilon, \quad 0<\varepsilon \leqslant 1 .
$$

Здесь $\mathscr{L}^{0}=\mathscr{L}\left(\eta^{0}, \mathbf{1}, 1\right)$. (См. ниже теорему 1.1.) В результате применения оценки (0.6) и получается аппроксимация (0.4).

Для оставшихся (пяти) полей в [4, гл. 7] получалась лишь слабая сходимость в $\mathfrak{G}$ к соответствующим «усредненным» полям.

0.3. В работе [10], [11] изучался общий случай переменных периодических коэффициентов $\eta(\mathbf{x}), \mu(\mathbf{x})$. Были получены равномерные аппроксимации по норме в $\mathfrak{G}$ для четырех полей из восъми, а именно для $\mathbf{v}_{\varepsilon}^{(r)}, \mathbf{z}_{\varepsilon}^{(r)}, \mathbf{u}_{\varepsilon}^{(q)}, \mathbf{w}_{\varepsilon}^{(q)}$, с точной по порядку оценкой погрешности. Помимо основного члена (решения усредненной системы) аппроксимации содержат слагаемые нулевого порядка, быстро осциллирующие при $\varepsilon \rightarrow 0$ (см. формулы $(2.45),(2.46),(2.49)$ ниже). Эти члены можно интерпретировать как корректоры нулевого порядка.

Для полей $\mathbf{v}_{\varepsilon}^{(r)}, \mathbf{z}_{\varepsilon}^{(r)}$ дело сводится к изучению оператора второго порядка

$$
\mathscr{L}_{\varepsilon}=\mathscr{L}\left(\eta^{\varepsilon}, \mu^{\varepsilon}, 1\right)=\left(\mu^{\varepsilon}\right)^{-1 / 2} \operatorname{rot}\left(\eta^{\varepsilon}\right)^{-1} \operatorname{rot}\left(\mu^{\varepsilon}\right)^{-1 / 2}-\left(\mu^{\varepsilon}\right)^{1 / 2} \nabla \operatorname{div}\left(\mu^{\varepsilon}\right)^{1 / 2},
$$

а для полей $\mathbf{u}_{\varepsilon}^{(q)}, \mathbf{w}_{\varepsilon}^{(q)}-$ к изучению аналогичного оператора $\mathscr{L}\left(\mu^{\varepsilon}, \eta^{\varepsilon}, 1\right)$. Оператор (0.7) (при переменном $\mu$ ) уже не входит в класс ДО, выделенный в [3], [4]. Однако к нему применимы результаты абстрактной теоретико-операторной схемы из [4, гл. 1]. На основе этой абстрактной схемы оператор (0.7) был изучен в [11], где для его резольвенты $\left(\mathscr{L}_{\varepsilon}+I\right)^{-1}$ была получена аппроксимация по операторной норме в $\mathfrak{G}$ с точной по порядку оценкой погрешности. Это позволило получить упомянутые аппроксимации для четырех полей.

0.4. В настоящей работе мы рассматриваем случай, когда магнитная проницаемость $\mu-$ постоянная положительная матрица: $\mu=\mu_{0}=$ Const. Для этого случая нам сейчас удалось получить равномерные аппроксимации по норме в $\mathfrak{G}$ для всех восъми полей (с оценкой погрешности порядка $\varepsilon$ ). Кроме того, для двух полей $\mathbf{v}_{\varepsilon}^{(r)}$ и $\mathbf{z}_{\varepsilon}^{(r)}$ получаются более точные аппроксимации по норме в $\mathfrak{G}$ (с оценкой погрешности порядка $\varepsilon^{2}$ ) и аппроксимации по норме в $\mathfrak{G}^{1}:=H^{1}\left(\mathbb{R}^{3} ; \mathbb{C}^{3}\right)$ с оценкой погрешности порядка $\varepsilon$. В этих аппроксимациях учитывается корректор первого порядка.

Мы опираемся не только на уже упоминавшиеся работы [4] и [10], [11], но и на недавние работы [6], [7], в которых задача об усреднении для класса ДО, выделенного в [3], [4], изучалась с учетом корректора.

Для полей $\mathbf{z}_{\varepsilon}^{(r)}, \mathbf{v}_{\varepsilon}^{(r)}$ вопрос сводится к изучению оператора $\mathscr{L}\left(\eta^{\varepsilon}, \mu_{0}, 1\right)$ (cр. $(0.7))$. Ниже в $§ 1$ мы рассматриваем несколько более общий оператор

$$
\mathscr{L}_{\varepsilon}=\mathscr{L}\left(\eta^{\varepsilon}, \mu_{0}, \nu^{\varepsilon}\right)=\mu_{0}^{-1 / 2} \operatorname{rot}\left(\eta^{\varepsilon}\right)^{-1} \operatorname{rot} \mu_{0}^{-1 / 2}-\mu_{0}^{1 / 2} \nabla \nu^{\varepsilon} \operatorname{div} \mu_{0}^{1 / 2}
$$

Здесь $\nu(\mathbf{x})$ есть Г-периодическая ограниченная положительная функция и $\nu^{\varepsilon}(\mathbf{x})$ $=\nu\left(\varepsilon^{-1} \mathbf{x}\right)$. Оператор (0.8) (при постоянном $\left.\mu_{0}\right)$ входит в класс ДО, выделенный в [3], [4]. Из общих результатов этих работ получается оценка вида $(0.6)$ для оператора (0.8). Сейчас $\mathscr{L}^{0}=\mathscr{L}\left(\eta^{0}, \mu_{0}, \underline{\nu}\right)$, где $\underline{\nu}^{-1}=|\Omega|^{-1} \int_{\Omega} \nu(\mathbf{x})^{-1} d \mathbf{x}$. 
Применение этого результата дает аппроксимации для полей $\mathbf{z}_{\varepsilon}^{(r)}, \mathbf{v}_{\varepsilon}^{(r)}$ через соответствующие «усредненные» поля:

$$
\left\|\mathbf{v}_{\varepsilon}^{(r)}-\mathbf{v}_{0}^{(r)}\right\|_{\mathfrak{G}} \leqslant C \varepsilon\|\mathbf{r}\|_{\mathfrak{G}}, \quad\left\|\mathbf{z}_{\varepsilon}^{(r)}-\mathbf{z}_{0}^{(r)}\right\|_{\mathfrak{G}} \leqslant C \varepsilon\|\mathbf{r}\|_{\mathfrak{G}}, \quad 0<\varepsilon \leqslant 1 .
$$

Далее, применяя общие результаты из [6], для резольвенты $\left(\mathscr{L}_{\varepsilon}+I\right)^{-1}$ получаем аппроксимацию по операторной норме в $\mathfrak{G}$ с оценкой погрешности порядка $\varepsilon^{2}$ (ср. 14 из [6], где рассматривался случай $\mu=\mathbf{1}$ ). В аппроксимации учитывается корректор первого порядка. Соответствующий результат сформулирован ниже в теореме 1.2. На этой основе получаются более точные аппроксимации в $\mathfrak{G}$ для полей $\mathbf{z}_{\varepsilon}^{(r)}, \mathbf{v}_{\varepsilon}^{(r)}$ (с погрешностью порядка $\left.\varepsilon^{2}\right)$.

Общие результаты работы [7] в применении к оператору (0.8) позволяют аппроксимировать резольвенту $\left(\mathscr{L}_{\varepsilon}+I\right)^{-1}$ по операторной норме из $\mathfrak{G}$ в $\mathfrak{G}^{1}$ с оценкой погрешности порядка $\varepsilon$ (ср. 222 из [7], где рассматривался случай $\mu=\mathbf{1})$. При этом учитывается корректор первого порядка. Соответствующий результат приведен ниже в теореме 1.3. Его применение дает аппроксимации для полей $\mathbf{z}_{\varepsilon}^{(r)}, \mathbf{v}_{\varepsilon}^{(r)}$ по норме в $\mathfrak{G}^{1}$ с погрешностью порядка $\varepsilon$.

Далее, поля $\mathbf{w}_{\varepsilon}^{(r)}, \mathbf{u}_{\varepsilon}^{(r)}$ выражаются через производные от $\mathbf{z}_{\varepsilon}^{(r)}$ :

$$
\mathbf{w}_{\varepsilon}^{(r)}=\operatorname{rot} \mu_{0}^{-1} \mathbf{z}_{\varepsilon}^{(r)}, \quad \mathbf{u}_{\varepsilon}^{(r)}=\left(\eta^{\varepsilon}\right)^{-1} \operatorname{rot} \mu_{0}^{-1} \mathbf{z}_{\varepsilon}^{(r)} .
$$

При этом $\mathbf{u}_{\varepsilon}^{(r)}$ играет роль так называемого «потока» для поля $\mathbf{z}_{\varepsilon}^{(r)}$. На основе результатов из [7] об аппроксимации потоков по норме в $\mathfrak{G}$ удается получить равномерные аппроксимации в $\mathfrak{G}$ для $\mathbf{u}_{\varepsilon}^{(r)}$ и $\mathbf{w}_{\varepsilon}^{(r)}$ (с погрешностью порядка $\varepsilon$ ). Эти аппроксимации (см. ниже (2.26), (2.27)) помимо соответствующих усредненных полей содержат слагаемые, быстро осциллирующие при $\varepsilon \rightarrow 0$. Эти слагаемые можно интерпретировать как корректоры нулевого порядка.

Что касается полей $\mathbf{u}_{\varepsilon}^{(q)}$ и $\mathbf{w}_{\varepsilon}^{(q)}$, то мы используем готовый результат из [10], [11], где для этих полей были получены равномерные аппроксимации в $\mathfrak{G}$ с погрешностью порядка $\varepsilon$. Наконец, результат для полей $\mathbf{v}_{\varepsilon}^{(q)}$ и $\mathbf{z}_{\varepsilon}^{(q)}$ получается на основе теоремы из [7] об аппроксимации «потоков» (см. ниже теорему 1.5), если перейти к сопряженным операторам.

Основные результаты работы приведены в теореме 2.1 (для случая $\mathbf{q}=0$ ) и в теореме 2.4 (для случая $\mathbf{r}=0$ ). Мы видим, что комбинация результатов работ [4], [10], [11], [6] и [7] позволяет в случае постоянного н полностью решить задачу об аппроксимащии всех физически интересных полей по норме в $\mathfrak{G}$ с погрешностъю порядка $\varepsilon$. Суммарный результат (аппроксимации в $\mathfrak{G}$ для четырех исходных полей $\left.\mathbf{u}_{\varepsilon}, \mathbf{v}_{\varepsilon}, \mathbf{w}_{\varepsilon}, \mathbf{z}_{\varepsilon}\right)$ приведен в теореме 2.7.

Разумеется, случай, когда коэффициент $\mu(\mathbf{x})$ периодичен, a $\eta=\eta_{0}=$ Const, вполне аналогичен рассмотренному. Мы остановились здесь на физически более естественном случае постоянного $\mu$.

0.5. В $\S 1$ приводятся (в удобном для дальнейшего виде) результаты из [4], [6], [7] о «модельном» операторе (0.8) второго порядка. При этом надо иметь в виду, что в указанных работах считалось, что $\mu=\mathbf{1}$. Однако случай $\mu=\mu_{0}$ рассматривается аналогично, и результаты $§ 1$ формулируются именно для этого случая.

Основные результаты об усреднении для оператора Максвелла собраны в 2 . 
0.6. По-видимому, разработанная авторами техника (см. [3]-[7], [10], [11]) открывает путь к получению аппроксимаций всех физических полей в общем случае переменных периодических проницаемостей $\eta(\mathbf{x}), \mu(\mathbf{x})$.

\section{§1. Модельный оператор второго порядка}

1.1. Определение оператора. Эффективный оператор. В этом параграфе мы рассматриваем вспомогательный оператор $\mathscr{L}$ второго порядка. Оператор $\mathscr{L}$ действует в пространстве $\mathfrak{G}=L_{2}\left(\mathbb{R}^{3} ; \mathbb{C}^{3}\right)$ и (формально) задается выражением

$$
\mathscr{L}=\mathscr{L}\left(\eta, \mu_{0}, \nu\right)=\mu_{0}^{-1 / 2} \operatorname{rot}(\eta(\mathbf{x}))^{-1} \operatorname{rot} \mu_{0}^{-1 / 2}-\mu_{0}^{1 / 2} \nabla \nu(\mathbf{x}) \operatorname{div} \mu_{0}^{1 / 2} .
$$

Здесь $\mu_{0}-$ постоянная положительно определенная $(3 \times 3)$-матрица с вещественными элементами, а $(3 \times 3)$-матрица-функция $\eta(\mathbf{x})$ с вещественными элементами и вещественная функция $\nu(\mathbf{x})$ периодичны относительно некоторой решетки $\Gamma \subset \mathbb{R}^{3}$, причем

$$
\begin{array}{ll}
\eta(\mathbf{x})>0, & \eta, \eta^{-1} \in L_{\infty}, \\
\nu(\mathbf{x})>0, & \nu, \nu^{-1} \in L_{\infty} .
\end{array}
$$

Точное определение оператора $\mathscr{L}$ дается через замкнутую в $\mathfrak{G}$ квадратичную форму

$\int_{\mathbb{R}^{3}}\left(\left\langle\eta(\mathbf{x})^{-1} \operatorname{rot} \mu_{0}^{-1 / 2} \mathbf{u}, \operatorname{rot} \mu_{0}^{-1 / 2} \mathbf{u}\right\rangle+\nu(\mathbf{x})\left|\operatorname{div} \mu_{0}^{1 / 2} \mathbf{u}\right|^{2}\right) d \mathbf{x}, \quad \mathbf{u} \in \mathfrak{G}^{1}=H^{1}\left(\mathbb{R}^{3} ; \mathbb{C}^{3}\right)$. Здесь $\langle\cdot, \cdot\rangle$ - стандартное скалярное произведение в $\mathbb{C}^{3}$. Для дальнейшего применения к системе Максвелла достаточно было бы считать, что $\nu(\mathbf{x})=1$.

Ниже через $\Omega$ обозначается ячейка решетки $\Gamma$, а через $\widetilde{\Omega}$ - центральная зона Бриллюена двойственной решетки. Далее, $\widetilde{H}^{1}\left(\Omega ; \mathbb{C}^{n}\right)-$ подпространство в классе Соболева $H^{1}\left(\Omega ; \mathbb{C}^{n}\right)$, состоящее из функций, Г-периодическое продолжение которых на $\mathbb{R}^{3}$ принадлежит классу $H_{\text {loc }}^{1}\left(\mathbb{R}^{3} ; \mathbb{C}^{n}\right)$. При $n=1$ пишем просто $\widetilde{H}^{1}(\Omega)$.

Оператор $\mathscr{L}$ принадлежит классу операторов, выделенному и изученному в работах [3], [4], [6], [7]. Он имеет вид $b(\mathbf{D})^{*} g(\mathbf{x}) b(\mathbf{D})$, где $g(\mathbf{x})$ - положительная $(4 \times 4)$-матрица, а $b(\mathbf{D})$ есть $(4 \times 3)$-матричный однородный дифференциальный оператор первого порядка. А именно,

$$
b(\mathbf{D})=\left(\begin{array}{c}
-i \operatorname{rot} \mu_{0}^{-1 / 2} \\
-i \operatorname{div} \mu_{0}^{1 / 2}
\end{array}\right), \quad g(\mathbf{x})=\left(\begin{array}{cc}
\eta(\mathbf{x})^{-1} & 0 \\
0 & \nu(\mathbf{x})
\end{array}\right) .
$$

В соответствии с общими правилами из [4] строится эффективный оператор $\mathscr{L}^{0}=b(\mathbf{D})^{*} g^{0} b(\mathbf{D})$. Эффективная матрица $g^{0}$ имеет вид

$$
g^{0}=\left(\begin{array}{cc}
\left(\eta^{0}\right)^{-1} & 0 \\
0 & \underline{\nu}
\end{array}\right)
$$

где $\eta^{0}$ - эффективная матрица для скалярного эллиптического оператора $-\operatorname{div} \eta \nabla=\mathbf{D}^{*} \eta \mathbf{D}$, а $\underline{\nu}$ определяется формулой

$$
(\underline{\nu})^{-1}=|\Omega|^{-1} \int_{\Omega} \nu(\mathbf{x})^{-1} d \mathbf{x} .
$$


Соответствующие вычисления при $\mu_{0}=\mathbf{1}$ проведены в $[4, \S 7.2]$.

Напомним, как определяется матрица $\eta^{0}$. Для каждого вектора $\mathbf{C} \in \mathbb{C}^{3}$ рассмотрим (слабое) Г-периодическое решение $\Phi_{\mathbf{C}} \in \widetilde{H}^{1}(\Omega)$ задачи

$$
\operatorname{div} \eta(\mathbf{x})\left(\nabla \Phi_{\mathbf{C}}(\mathbf{x})+\mathbf{C}\right)=0, \quad \int_{\Omega} \Phi(\mathbf{x}) d \mathbf{x}=0 .
$$

Тогда постоянная матрица $\eta^{0}$ определяется соотношением

$$
\eta^{0} \mathbf{C}=|\Omega|^{-1} \int_{\Omega} \eta(\mathbf{x})\left(\nabla \Phi_{\mathbf{C}}(\mathbf{x})+\mathbf{C}\right) d \mathbf{x}, \quad \mathbf{C} \in \mathbb{C}^{3} .
$$

Эффективный оператор $\mathscr{L}^{0}$ действует в $\mathfrak{G}$ по формуле

$$
\mathscr{L}^{0}=\mathscr{L}\left(\eta^{0}, \mu_{0}, \underline{\nu}\right)=\mu_{0}^{-1 / 2} \operatorname{rot}\left(\eta^{0}\right)^{-1} \operatorname{rot} \mu_{0}^{-1 / 2}-\mu_{0}^{1 / 2} \nabla \underline{\nu} \operatorname{div} \mu_{0}^{1 / 2} .
$$

1.2. Оператор $\mathscr{L}_{\varepsilon}$. Старший член аппроксимации резольвенты $\left(\mathscr{L}_{\varepsilon}+I\right)^{-1}$ по операторной $\left(L_{2}\right)$-норме. Условимся для произвольной $\Gamma$-периодической функции $\phi(\mathbf{x})$ использовать обозначение $\phi^{\varepsilon}(\mathbf{x})=\phi\left(\varepsilon^{-1} \mathbf{x}\right), \varepsilon>0$. Рассмотрим оператор

$$
\mathscr{L}_{\varepsilon}=\mathscr{L}\left(\eta^{\varepsilon}, \mu_{0}, \nu^{\varepsilon}\right)=\mu_{0}^{-1 / 2} \operatorname{rot}\left(\eta^{\varepsilon}\right)^{-1} \operatorname{rot} \mu_{0}^{-1 / 2}-\mu_{0}^{1 / 2} \nabla \nu^{\varepsilon} \operatorname{div} \mu_{0}^{1 / 2} .
$$

Коэффициенты оператора (1.7) быстро осциллируют при $\varepsilon \rightarrow 0$. Задача усреднения для этого оператора состоит в аппроксимации резольвенты $\left(\mathscr{L}_{\varepsilon}+I\right)^{-1}$ при малом $\varepsilon$. Следующая теорема дает старший член аппроксимации по операторной норме в $\mathfrak{G}$. Этот результат получается применением общей теоремы 4.2.1 из [4] (ср. с теоремой 7.2.1 из [4], где рассмотрен случай $\mu_{0}=\mathbf{1}$ ).

Теорема 1.1. Пусть выполнены условия (1.2), (1.3). Пусть $\mathscr{L}_{\varepsilon}$ - оператор (1.7) и $\mathscr{L}^{0}$ - эффективный оператор (1.6). Тогда справедлива оченка

$$
\left\|\left(\mathscr{L}_{\varepsilon}+I\right)^{-1}-\left(\mathscr{L}^{0}+I\right)^{-1}\right\|_{\mathfrak{G} \rightarrow \mathfrak{G}} \leqslant C_{1} \varepsilon, \quad 0<\varepsilon \leqslant 1 .
$$

Постоянная $C_{1}$ зависит лишь от матричных норм $\left|\mu_{0}\right|,\left|\mu_{0}^{-1}\right|$, от норм коэбфичиентов $\|\eta\|_{L_{\infty}},\left\|\eta^{-1}\right\|_{L_{\infty}},\|\nu\|_{L_{\infty}},\left\|\nu^{-1}\right\|_{L_{\infty}}$ и от параметров решетки $\Gamma$.

Отметим, что оценка (1.8) - точная по порядку, а постоянная $C_{1}$ допускает явный контроль.

1.3. Аппроксимация резольвенты $\left(\mathscr{L}_{\varepsilon}+I\right)^{-1}$ по операторной $\left(L_{2}\right)$ норме с учетом корректора. К оператору (1.7) применима общая теорема 8.2 из [6], в которой дается аппроксимация резольвенты по операторной норме в $\mathfrak{G}$ с оценкой погрешности порядка $\varepsilon^{2}$. В аппроксимации учитывается корректор. Корректор вычисляется по общим правилам, приведенным в [6], он содержит три члена. При $\mu_{0}=1$ корректор для оператора (1.7) вычислен в $[6, \S 14] ;$ эти вычисления без труда переносятся на случай произвольной постоянной матрицы $\mu_{0}$. Введем объекты, необходимые для описания корректора. Пусть $\mathbf{e}_{j}, j=$ $1,2,3$, - стандартные орты в $\mathbb{C}^{3}$. Положим $\mathbf{c}_{j}=\left(\eta^{0}\right)^{-1} \mathbf{e}_{j}, j=1,2,3$. Пусть $\widetilde{\Phi}_{j}=\Phi_{\mathbf{c}_{j}}-$ периодическое решение задачи (1.4) при $\mathbf{C}=\mathbf{c}_{j}$. Далее, пусть $\mathbf{p}_{j} \in \widetilde{H}^{1}\left(\Omega ; \mathbb{C}^{3}\right)$ есть $Г$-периодическое решение задачи

$$
\operatorname{rot} \mu_{0}^{-1} \operatorname{rot} \mathbf{p}_{j}=\eta\left(\nabla \widetilde{\Phi}_{j}+\mathbf{c}_{j}\right)-\mathbf{e}_{j}, \quad \operatorname{div} \mathbf{p}_{j}=0, \quad \int_{\Omega} \mathbf{p}_{j} d \mathbf{x}=0 .
$$


Обозначим через $\Psi(\mathbf{x})$ вещественную $(3 \times 3)$-матрицу со столбцами rot $\mathbf{p}_{1}$, $\operatorname{rot} \mathbf{p}_{2}, \operatorname{rot} \mathbf{p}_{3}$. Положим

$$
\alpha_{l j s}=|\Omega|^{-1} \int_{\Omega} \widetilde{\Phi}_{l}(\mathbf{x})\left\langle\eta(\mathbf{x})\left(\nabla \widetilde{\Phi}_{j}(\mathbf{x})+\mathbf{c}_{j}\right), \mathbf{e}_{s}\right\rangle d \mathbf{x}, \quad l, j, s=1,2,3,
$$

и введем скалярный (самосопряженный) оператор второго порядка

$$
\mathscr{E}(\mathbf{D})=\sum_{s=1}^{3}\left(\left(\alpha_{12 s}-\alpha_{21 s}\right) \partial_{3} \partial_{s}+\left(\alpha_{31 s}-\alpha_{13 s}\right) \partial_{2} \partial_{s}+\left(\alpha_{23 s}-\alpha_{32 s}\right) \partial_{1} \partial_{s}\right)
$$

Далее, пусть $\varphi \in \widetilde{H}^{1}(\Omega)-$ решение задачи

$$
\operatorname{div} \mu_{0} \nabla \varphi=\underline{\nu}(\nu(\mathbf{x}))^{-1}-1, \quad \int_{\Omega} \varphi d \mathbf{x}=0 .
$$

Положим $\mathbf{b}=\nabla \varphi$.

Корректор $K(\varepsilon)$ имеет вид (ср. с формулой (14.15) из [6])

$$
\begin{aligned}
K(\varepsilon)=( & \left.\mu_{0}^{-1 / 2} \Psi^{\varepsilon} \operatorname{rot} \mu_{0}^{-1 / 2}+\mu_{0}^{1 / 2} \mathbf{b}^{\varepsilon} \operatorname{div} \mu_{0}^{1 / 2}\right)\left(\mathscr{L}^{0}+I\right)^{-1} \\
& +\left(\mathscr{L}^{0}+I\right)^{-1}\left(\mu_{0}^{-1 / 2} \operatorname{rot}\left(\Psi^{\varepsilon}\right)^{t} \mu_{0}^{-1 / 2}-\mu_{0}^{1 / 2} \nabla\left(\mathbf{b}^{\varepsilon}\right)^{t} \mu_{0}^{1 / 2}\right) \\
& -\left(\mathscr{L}^{0}+I\right)^{-1} \mu_{0}^{-1 / 2} \mathscr{E}(\mathbf{D}) \operatorname{rot} \mu_{0}^{-1 / 2}\left(\mathscr{L}^{0}+I\right)^{-1} .
\end{aligned}
$$

Здесь и ниже верхний индекс $t$ означает операцию транспонирования матрицы. Корректор (1.12) не содержит вспомогательного сглаживающего оператора, который необходимо включать в корректор в общем случае. Действительно, у нас размерность $d=3$, т. е. выполнено условие 6.10(1) из [6] (условие $d \leqslant 4$ ), при котором можно избавиться от сглаживателя в корректоре. Отметим, что первые два слагаемых справа в (1.12) взаимно сопряжены, а третье не зависит от $\varepsilon$.

Применяя теорему 8.2 из [6], приходим к следующему результату (ср. с теоремой 14.1 из [6]).

Теорема 1.2. Пусть $\mathscr{L}_{\varepsilon}$ - оператор (1.7) и $\mathscr{L}^{0}$ - эффективный оператор (1.6). Пусть оператор $K(\varepsilon)$ определен формулой (1.12). Тогда выполнена оценка

$$
\left\|\left(\mathscr{L}_{\varepsilon}+I\right)^{-1}-\left(\mathscr{L}^{0}+I\right)^{-1}-\varepsilon K(\varepsilon)\right\|_{\mathfrak{G} \rightarrow \mathfrak{G}} \leqslant C_{2} \varepsilon^{2}, \quad 0<\varepsilon \leqslant 1 .
$$

Постоянная $C_{2}$ зависит лишь от матричных норм $\left|\mu_{0}\right|,\left|\mu_{0}^{-1}\right|$, от норм коэффичиентов $\|\eta\|_{L_{\infty}},\left\|\eta^{-1}\right\|_{L_{\infty}},\|\nu\|_{L_{\infty}},\left\|\nu^{-1}\right\|_{L_{\infty}}$ и от параметров решетки $\Gamma$.

\section{4. Аппроксимация резольвенты $\left(\mathscr{L}_{\varepsilon}+I\right)^{-1}$ по операторной нор-} ме из $\boldsymbol{L}_{\mathbf{2}}$ в $\boldsymbol{H}^{\mathbf{1}}$ с учетом корректора. K оператору (1.7) применима общая теорема 10.6 из [7], которая дает аппроксимацию резольвенты по операторной норме из $\mathfrak{G}$ в $\mathfrak{G}^{1}=H^{1}\left(\mathbb{R}^{3} ; \mathbb{C}^{3}\right)$ с оценкой погрешности порядка $\varepsilon$. В аппроксимацию включается корректор $\widetilde{K}_{1}(\varepsilon)$, который отвечает первому слагаемому из (1.12). Сейчас, однако, в корректор необходимо включить сглаживающий оператор $\Pi_{\varepsilon}$. (При аппроксимации резольвенты по операторной норме из $L_{2}$ в $H^{1}$ условия, при которых удается избавиться от сглаживателя, жестче, чем при аппроксимациях по операторной норме из $L_{2}$ в $L_{2}$.) Оператор $\Pi_{\varepsilon}$ представляет собой ПДО (псевдодифференциальный оператор) в $\mathfrak{G}$ с символом $\chi_{\widetilde{\Omega} / \varepsilon}(\boldsymbol{\xi})$, где $\chi_{\widetilde{\Omega} / \varepsilon}(\boldsymbol{\xi})-$ характеристическая функция множества $\widetilde{\Omega} / \varepsilon$. Положим

$$
\widetilde{K}_{1}(\varepsilon)=\left(\mu_{0}^{-1 / 2} \Psi^{\varepsilon} \operatorname{rot} \mu_{0}^{-1 / 2}+\mu_{0}^{1 / 2} \mathbf{b}^{\varepsilon} \operatorname{div} \mu_{0}^{1 / 2}\right)\left(\mathscr{L}^{0}+I\right)^{-1} \Pi_{\varepsilon} .
$$


Применяя теорему 10.6 из [7], получаем следующий результат (ср. с теоремой 22.1 из [7], где был рассмотрен случай $\mu_{0}=\mathbf{1}$ ).

Теорема 1.3. Пусть $\mathscr{L}_{\varepsilon}$ - оператор (1.7) и $\mathscr{L}^{0}$ - эфбективный оператор (1.6). Пусть оператор $\widetilde{K}_{1}(\varepsilon)$ определен в (1.14). Тогда выполнена оценка

$$
\left\|\left(\mathscr{L}_{\varepsilon}+I\right)^{-1}-\left(\mathscr{L}^{0}+I\right)^{-1}-\varepsilon \widetilde{K}_{1}(\varepsilon)\right\|_{\mathfrak{G} \rightarrow \mathfrak{G}^{1}} \leqslant C_{3} \varepsilon, \quad 0<\varepsilon \leqslant 1 .
$$

Постоянная $C_{3}$ зависит лишь от $\left|\mu_{0}\right|,\left|\mu_{0}^{-1}\right|,\|\eta\|_{L_{\infty}},\left\|\eta^{-1}\right\|_{L_{\infty}},\|\nu\|_{L_{\infty}},\left\|\nu^{-1}\right\|_{L_{\infty}}$ и от параметров решетки Г.

1.5. Интерполяционные результаты. K оператору (1.7) применима также (интерполяционная) теорема 11.3 из [7], в которой устанавливается аппроксимация резольвенты по операторной норме из $\mathfrak{G}$ в $\mathfrak{G}^{s}=H^{s}\left(\mathbb{R}^{3} ; \mathbb{C}^{3}\right)$ при $0<s \leqslant 1$. В этой аппроксимации используется трехчленный корректор $\widetilde{K}(\varepsilon)$, который отличается от корректора (1.12) наличием сглаживателя в первых двух членах:

$$
\begin{aligned}
\widetilde{K}(\varepsilon)=( & \left.\mu_{0}^{-1 / 2} \Psi^{\varepsilon} \operatorname{rot} \mu_{0}^{-1 / 2}+\mu_{0}^{1 / 2} \mathbf{b}^{\varepsilon} \operatorname{div} \mu_{0}^{1 / 2}\right)\left(\mathscr{L}^{0}+I\right)^{-1} \Pi_{\varepsilon} \\
& +\left(\mathscr{L}^{0}+I\right)^{-1} \Pi_{\varepsilon}\left(\mu_{0}^{-1 / 2} \operatorname{rot}\left(\Psi^{\varepsilon}\right)^{t} \mu_{0}^{-1 / 2}-\mu_{0}^{1 / 2} \nabla\left(\mathbf{b}^{\varepsilon}\right)^{t} \mu_{0}^{1 / 2}\right) \\
& -\left(\mathscr{L}^{0}+I\right)^{-1} \mu_{0}^{-1 / 2} \mathscr{E}(\mathbf{D}) \operatorname{rot} \mu_{0}^{-1 / 2}\left(\mathscr{L}^{0}+I\right)^{-1} .
\end{aligned}
$$

Теорема 1.4. Пусть $\mathscr{L}_{\varepsilon}$ - оператор (1.7) и $\mathscr{L}^{0}-$ эфбективный оператор (1.6). Пусть оператор $\widetilde{K}(\varepsilon)$ определен формулой (1.16). Тогда при $0<s \leqslant 1$ выполнена оченка

$$
\left\|\left(\mathscr{L}_{\varepsilon}+I\right)^{-1}-\left(\mathscr{L}^{0}+I\right)^{-1}-\varepsilon \widetilde{K}(\varepsilon)\right\|_{\mathfrak{G} \rightarrow \mathfrak{G}^{s}} \leqslant \mathscr{C}_{s} \varepsilon^{2-s}, \quad 0<\varepsilon \leqslant 1 .
$$

Постоянная $\mathscr{C}_{s}$ зависит лишь от $s,\left|\mu_{0}\right|,\left|\mu_{0}^{-1}\right|,\|\eta\|_{L_{\infty}},\left\|\eta^{-1}\right\|_{L_{\infty}},\|\nu\|_{L_{\infty}}$, $\left\|\nu^{-1}\right\|_{L_{\infty}}$ и от параметров решетки $\Gamma$.

1.6. Аппроксимация потоков в $\boldsymbol{L}_{2}$. Пусть $\mathbf{f}_{\varepsilon}-$ решение уравнения

$$
\mathscr{L}_{\varepsilon} \mathbf{f}_{\varepsilon}+\mathbf{f}_{\varepsilon}=\mathbf{F}, \quad \mathbf{F} \in \mathfrak{G},
$$

и $\mathbf{f}_{0}$ - решение усредненного уравнения

$$
\mathscr{L}^{0} \mathbf{f}_{0}+\mathbf{f}_{0}=\mathbf{F} .
$$

Оценка (1.15) означает, что

$$
\left\|\mathbf{f}_{\varepsilon}-\mathbf{f}_{0}-\varepsilon \widetilde{K}_{1}(\varepsilon) \mathbf{F}\right\|_{\mathfrak{G}^{1}} \leqslant C_{3} \varepsilon\|\mathbf{F}\|_{\mathfrak{G},}, \quad 0<\varepsilon \leqslant 1 .
$$

Отметим, что слабый $\left(\mathfrak{G}^{1}\right)$-предел функций $\mathbf{f}_{\varepsilon}$ при $\varepsilon \rightarrow 0$ равен $\mathbf{f}_{0}$ (см. замечание 10.7 из [7]).

Роль потока для уравнения (1.18) играет вектор-функция

$$
g^{\varepsilon} b(\mathbf{D}) \mathbf{f}_{\varepsilon}=-i\left(\begin{array}{c}
\left(\eta^{\varepsilon}\right)^{-1} \operatorname{rot} \mu_{0}^{-1 / 2} \mathbf{f}_{\varepsilon} \\
\nu^{\varepsilon} \operatorname{div} \mu_{0}^{1 / 2} \mathbf{f}_{\varepsilon}
\end{array}\right) .
$$

Через $a(\mathbf{x})$ обозначим $(3 \times 3)$-матрицу со столбцами $\left(\nabla \widetilde{\Phi}_{j}+\mathbf{c}_{j}\right), j=1,2,3$. Применяя теорему 12.1 из [7] об аппроксимации потоков, получаем следующие 
две оценки (ср. с оценками $(22.16),(22.17)$ из [7], где рассмотрен случай $\left.\mu_{0}=1\right)$ :

$$
\begin{aligned}
\left\|\left(\eta^{\varepsilon}\right)^{-1} \operatorname{rot} \mu_{0}^{-1 / 2} \mathbf{f}_{\varepsilon}-a^{\varepsilon} \Pi_{\varepsilon} \operatorname{rot} \mu_{0}^{-1 / 2} \mathbf{f}_{0}\right\|_{\mathfrak{G}} \leqslant C_{4} \varepsilon\|\mathbf{F}\|_{\mathfrak{G},} & 0<\varepsilon \leqslant 1, \\
\left\|\nu^{\varepsilon} \operatorname{div} \mathbf{f}_{\varepsilon}-\underline{\nu} \Pi_{\varepsilon}^{(1)} \operatorname{div} \mathbf{f}_{0}\right\|_{L_{2}\left(\mathbb{R}^{3}\right)} \leqslant C_{4} \varepsilon\|\mathbf{F}\|_{\mathfrak{G},}, & 0<\varepsilon \leqslant 1 .
\end{aligned}
$$

Здесь $\Pi_{\varepsilon}^{(1)}$ есть ПДО в $L_{2}\left(\mathbb{R}^{3}\right)$ с символом $\chi_{\widetilde{\Omega} / \varepsilon}(\boldsymbol{\xi})$.

Аналогично тому, как это сделано в п. 22.3 из [7], нетрудно показать, что сглаживатели $\Pi_{\varepsilon}, \Pi_{\varepsilon}^{(1)}$ в оценках $(1.20),(1.21)$ можно заменить на $I$; такая замена отразится лишь на постоянных в оценках. При этом «устранение» сглаживателя в (1.21) тривиально, а в (1.20) основано на том, что столбцы матрицы $a(\mathbf{x})$ (которые выражаются через градиенты решений $\widetilde{\Phi}_{j}$ задачи вида $\left.(1.4)\right)$ являются мультипликаторами из $H^{1}$ в $L_{2}$. Последнее утверждение было установлено в [11, предложение 8.2].

Отметим, что $a(\mathbf{x})=(\mathbf{1}+Z(\mathbf{x}))\left(\eta^{0}\right)^{-1}$, где $Z(\mathbf{x})-($ вещественная) матрица со столбцами $\nabla \Phi_{j}(\mathbf{x}), j=1,2,3$, и $\Phi_{j}=\Phi_{\mathbf{e}_{j}}-$ решение уравнения (1.4) при $\mathbf{C}=\mathbf{e}_{j}$. В итоге приходим к следующему утверждению.

Теорема 1.5. Пусть выполнены условия теоремы 1.1. Пусть $\mathbf{f}_{\varepsilon}$ - решение уравнения (1.18), а $\mathbf{f}_{0}$ - решение усредненного уравнения (1.19). Пусть $Z(\mathbf{x})$ есть $(3 \times 3)$-матрица со столбиами $\nabla \Phi_{j}(\mathbf{x}), j=1,2,3$, где $\Phi_{j}$ является Г-периодическим решением задачи (1.4) при $\mathbf{C}=\mathbf{e}_{j}$. Тогда справедливы оценки

$$
\left\|\left(\eta^{\varepsilon}\right)^{-1} \operatorname{rot} \mu_{0}^{-1 / 2} \mathbf{f}_{\varepsilon}-\left(\mathbf{1}+Z^{\varepsilon}\right)\left(\eta^{0}\right)^{-1} \operatorname{rot} \mu_{0}^{-1 / 2} \mathbf{f}_{0}\right\|_{\mathfrak{G}} \leqslant C_{5} \varepsilon\|\mathbf{F}\|_{\mathfrak{G},}, \quad 0<\varepsilon \leqslant 1,
$$

$$
\left\|\nu^{\varepsilon} \operatorname{div} \mathbf{f}_{\varepsilon}-\underline{\nu} \operatorname{div} \mathbf{f}_{0}\right\|_{L_{2}\left(\mathbb{R}^{3}\right)} \leqslant C_{6} \varepsilon\|\mathbf{F}\|_{\mathfrak{G}}, \quad 0<\varepsilon \leqslant 1 .
$$

Постоянные $C_{5}, C_{6}$ зависят лишь от $\left|\mu_{0}\right|,\left|\mu_{0}^{-1}\right|,\|\eta\|_{L_{\infty}},\left\|\eta^{-1}\right\|_{L_{\infty}},\|\nu\|_{L_{\infty}}$, $\left\|\nu^{-1}\right\|_{L_{\infty}}$ и от параметров решетки $\Gamma$.

Отметим, что при $\varepsilon \rightarrow 0$ вектор-функции $\left(\eta^{\varepsilon}\right)^{-1} \operatorname{rot} \mu_{0}^{-1 / 2} \mathbf{f}_{\varepsilon}$ слабо сходятся в $\mathfrak{G}$ к вектору $\left(\eta^{0}\right)^{-1} \operatorname{rot} \mu_{0}^{-1 / 2} \mathbf{f}_{0}$ (см. замечание 12.2 из [7]).

1.7. Особые случаи. Положим

$$
\bar{g}=|\Omega|^{-1} \int_{\Omega} g(\mathbf{x}) d \mathbf{x}, \quad(\underline{g})^{-1}=|\Omega|^{-1} \int_{\Omega} g(\mathbf{x})^{-1} d \mathbf{x} .
$$

Для эффективной матрицы $g^{0}$ всегда выполнены неравенства $\underline{g} \leqslant g^{0} \leqslant \bar{g}$. В случае когда $g^{0}=\bar{g}$, корректоры $(1.12),(1.14),(1.16)$ обращаются в нуль. Этот случай реализуется, если $\nu=$ Const и $\eta^{0}=\eta$, т. е. столбцы матрицы $\eta^{-1}-$ потенциальные векторы (см. предложение 3.1 .7 из [4]). В этом случае применимы теорема 8.3 из [6] и теоремы 10.9 и 11.5 из [7], которые дают следующий результат.

Теорема 1.6. Пусть выполнены условия теоремы 1.1. Пусть, кроме того, $\nu=$ Const $u \eta^{0}=\underline{\eta}$. Тогда при $0<\varepsilon \leqslant 1$ справедливы оценки

$$
\begin{aligned}
& \left\|\left(\mathscr{L}_{\varepsilon}+I\right)^{-1}-\left(\mathscr{L}^{0}+I\right)^{-1}\right\|_{\mathfrak{G} \rightarrow \mathfrak{G}} \leqslant \widetilde{C}_{2} \varepsilon^{2}, \\
& \left\|\left(\mathscr{L}_{\varepsilon}+I\right)^{-1}-\left(\mathscr{L}^{0}+I\right)^{-1}\right\|_{\mathfrak{G} \rightarrow \mathfrak{G}^{1}} \leqslant \widetilde{C}_{3} \varepsilon, \\
& \left\|\left(\mathscr{L}_{\varepsilon}+I\right)^{-1}-\left(\mathscr{L}^{0}+I\right)^{-1}\right\|_{\mathfrak{G} \rightarrow \mathfrak{G}^{s}} \leqslant \widetilde{\mathscr{C}}_{s} \varepsilon^{2-s}, \quad 0<s \leqslant 1 .
\end{aligned}
$$


Постоянные $\widetilde{C}_{2}, \widetilde{C}_{3}$ зависят от $\left|\mu_{0}\right|,\left|\mu_{0}^{-1}\right|,\|\eta\|_{L_{\infty}},\left\|\eta^{-1}\right\|_{L_{\infty}},\|\nu\|_{L_{\infty}},\left\|\nu^{-1}\right\|_{L_{\infty}}$ и от параметров решетки $\Gamma$, а $\widetilde{\mathscr{C}}_{s}$ зависит от тех же параметров и от $\mathrm{s}$.

Случай $g^{0}=\underline{g}$ реализуется при произвольном $\nu(\mathbf{x})$ и при $\eta^{0}=\bar{\eta}$ (т. е. если столбцы матрицы $\eta(\mathbf{x})$ соленоидальны; см. предложение 3.1.6 из [4]). В этом случае $Z(\mathbf{x})=0$, а потому формула (1.22) упрощается (ср. с теоремой 12.4 из [7]). Сформулируем результат.

Теорема 1.7. Пусть выполнены условия теоремы 1.5. Пусть, кроме того, $\eta^{0}=\bar{\eta}$. Тогда при $0<\varepsilon \leqslant 1$ выполнена оценка

$$
\left\|\left(\eta^{\varepsilon}\right)^{-1} \operatorname{rot} \mu_{0}^{-1 / 2} \mathbf{f}_{\varepsilon}-(\bar{\eta})^{-1} \operatorname{rot} \mu_{0}^{-1 / 2} \mathbf{f}_{0}\right\|_{\mathfrak{G}} \leqslant C_{5} \varepsilon\|\mathbf{F}\|_{\mathfrak{G}} .
$$

\section{8. Распадение оператора $\mathscr{L}$. Положим}

$$
\begin{gathered}
J\left(\mu_{0}\right)=\left\{\mathbf{f} \in \mathfrak{G}: \operatorname{div} \mu_{0}^{1 / 2} \mathbf{f}=0\right\}, \\
G\left(\mu_{0}\right)=\left\{\mathbf{u}=\mu_{0}^{1 / 2} \nabla \phi: \phi \in H_{\text {loc }}^{1}\left(\mathbb{R}^{3}\right), \nabla \phi \in \mathfrak{G}\right\} .
\end{gathered}
$$

Справедливо ортогональное разложение Вейля

$$
\mathfrak{G}=L_{2}\left(\mathbb{R}^{3} ; \mathbb{C}^{3}\right)=J\left(\mu_{0}\right) \oplus G\left(\mu_{0}\right) .
$$

Разложение (1.28) приводит оператор (1.1): $\mathscr{L}=\mathscr{L}_{J} \oplus \mathscr{L}_{G}$. Оператор $\mathscr{L}_{J}$, действующий в подпространстве $J\left(\mu_{0}\right)$, соответствует дифференциальному выражению $\mu_{0}^{-1 / 2} \operatorname{rot} \eta^{-1} \operatorname{rot} \mu_{0}^{-1 / 2}$, а оператор $\mathscr{L}_{G}$, действующий в $G\left(\mu_{0}\right)$, задается выражением $-\mu_{0}^{1 / 2} \nabla \nu \operatorname{div} \mu_{0}^{1 / 2}$. Операторы $\mathscr{L}_{\varepsilon}$ и $\mathscr{L}^{0}$ также приводятся разложением (1.28): $\mathscr{L}_{\varepsilon}=\mathscr{L}_{J, \varepsilon} \oplus \mathscr{L}_{G, \varepsilon}, \mathscr{L}^{0}=\mathscr{L}_{J}^{0} \oplus \mathscr{L}_{G}^{0}$. Для нас (для применений к системе Максвелла) представляют интерес операторы $\mathscr{L}_{J}, \mathscr{L}_{J, \varepsilon}$ и $\mathscr{L}_{J}^{0}$. Поскольку они не зависят от коэффициента $\nu$, достаточно рассмотреть случай, когда $\nu=1$.

Пусть $\mathscr{P}\left(\mu_{0}\right)$ - ортопроектор в $\mathfrak{G}$ на $J\left(\mu_{0}\right)$. Тогда (ср. [4, п. 2.4 гл. 7]) оператор $\mathscr{P}\left(\mu_{0}\right)$ (суженный на $\left.\mathfrak{G}^{s}\right)$ является ортопроектором и в пространстве $\mathfrak{G}^{s}=H^{s}\left(\mathbb{R}^{3} ; \mathbb{C}^{3}\right)$ на подпространство $J^{s}\left(\mu_{0}\right)=\mathfrak{G}^{s} \cap J\left(\mu_{0}\right)$ при всех $s>0$.

Сужая операторы в $(1.8)$ на подпространство $J\left(\mu_{0}\right)$, получаем неравенство

$$
\left\|\left(\mathscr{L}_{J, \varepsilon}+I_{J\left(\mu_{0}\right)}\right)^{-1}-\left(\mathscr{L}_{J}^{0}+I_{J\left(\mu_{0}\right)}\right)^{-1}\right\|_{J\left(\mu_{0}\right) \rightarrow J\left(\mu_{0}\right)} \leqslant C_{1}^{\prime} \varepsilon, \quad 0<\varepsilon \leqslant 1 .
$$

Постоянная $C_{1}^{\prime}$ есть $C_{1}$ при $\nu=1$, а потому $C_{1}^{\prime}$ зависит лишь от $\left|\mu_{0}\right|,\left|\mu_{0}^{-1}\right|$, $\|\eta\|_{L_{\infty}},\left\|\eta^{-1}\right\|_{L_{\infty}}$ и от параметров решетки $\Gamma$.

Сужая операторы в $(1.13)$ на $J\left(\mu_{0}\right)$ и домножая их слева на $\mathscr{P}\left(\mu_{0}\right)$, имеем

$$
\left\|\left(\mathscr{L}_{J, \varepsilon}+I_{J\left(\mu_{0}\right)}\right)^{-1}-\left(\mathscr{L}_{J}^{0}+I_{J\left(\mu_{0}\right)}\right)^{-1}-\varepsilon K_{J}(\varepsilon)\right\|_{J\left(\mu_{0}\right) \rightarrow J\left(\mu_{0}\right)} \leqslant C_{2}^{\prime} \varepsilon^{2}, \quad 0<\varepsilon \leqslant 1,
$$

где $C_{2}^{\prime}$ есть $C_{2}$ при $\nu=1$ и

$$
\begin{aligned}
K_{J}(\varepsilon)= & \mathscr{P}\left(\mu_{0}\right) \mu_{0}^{-1 / 2} \Psi^{\varepsilon} \operatorname{rot} \mu_{0}^{-1 / 2}\left(\mathscr{L}_{J}^{0}+I_{J\left(\mu_{0}\right)}\right)^{-1} \\
& +\left(\mathscr{L}_{J}^{0}+I_{J\left(\mu_{0}\right)}\right)^{-1} \mu_{0}^{-1 / 2} \operatorname{rot}\left(\Psi^{\varepsilon}\right)^{t} \mu_{0}^{-1 / 2} \\
& -\left(\mathscr{L}_{J}^{0}+I_{J\left(\mu_{0}\right)}\right)^{-1} \mu_{0}^{-1 / 2} \mathscr{E}(\mathbf{D}) \operatorname{rot} \mu_{0}^{-1 / 2}\left(\mathscr{L}_{J}^{0}+I_{J\left(\mu_{0}\right)}\right)^{-1} .
\end{aligned}
$$


Аналогично, сужая операторы в $(1.15)$ на $J\left(\mu_{0}\right)$ и домножая на $\mathscr{P}\left(\mu_{0}\right)$, получаем

$$
\left\|\left(\mathscr{L}_{J, \varepsilon}+I_{J\left(\mu_{0}\right)}\right)^{-1}-\left(\mathscr{L}_{J}^{0}+I_{J\left(\mu_{0}\right)}\right)^{-1}-\varepsilon \widetilde{K}_{1, J}(\varepsilon)\right\|_{J\left(\mu_{0}\right) \rightarrow J^{1}\left(\mu_{0}\right)} \leqslant C_{3}^{\prime} \varepsilon, \quad 0<\varepsilon \leqslant 1,
$$

где $C_{3}^{\prime}$ есть $C_{3}$ при $\nu=1$ и

$$
\widetilde{K}_{1, J}(\varepsilon)=\mathscr{P}\left(\mu_{0}\right) \mu_{0}^{-1 / 2} \Psi^{\varepsilon} \Pi_{\varepsilon} \operatorname{rot} \mu_{0}^{-1 / 2}\left(\mathscr{L}_{J}^{0}+I_{J\left(\mu_{0}\right)}\right)^{-1} .
$$

Наконец, из неравенства (1.17) выводится оценка

$$
\begin{gathered}
\left\|\left(\mathscr{L}_{J, \varepsilon}+I_{J\left(\mu_{0}\right)}\right)^{-1}-\left(\mathscr{L}_{J}^{0}+I_{J\left(\mu_{0}\right)}\right)^{-1}-\varepsilon \widetilde{K}_{J}(\varepsilon)\right\|_{J\left(\mu_{0}\right) \rightarrow J^{s}\left(\mu_{0}\right)} \leqslant \mathscr{C}_{s}^{\prime} \varepsilon^{2-s}, \\
0<s \leqslant 1,0<\varepsilon \leqslant 1,
\end{gathered}
$$

где $\mathscr{C}_{s}^{\prime}$ есть $\mathscr{C}_{s}$ при $\nu=1$ и

$$
\begin{aligned}
& \widetilde{K}_{J}(\varepsilon)= \mathscr{P} \\
&\left(\mu_{0}\right) \mu_{0}^{-1 / 2} \Psi^{\varepsilon} \operatorname{rot} \mu_{0}^{-1 / 2}\left(\mathscr{L}_{J}^{0}+I_{J\left(\mu_{0}\right)}\right)^{-1} \Pi_{\varepsilon} \\
&+\left(\mathscr{L}_{J}^{0}+I_{J\left(\mu_{0}\right)}\right)^{-1} \Pi_{\varepsilon} \mu_{0}^{-1 / 2} \operatorname{rot}\left(\Psi^{\varepsilon}\right)^{t} \mu_{0}^{-1 / 2} \\
&-\left(\mathscr{L}_{J}^{0}+I_{J\left(\mu_{0}\right)}\right)^{-1} \mu_{0}^{-1 / 2} \mathscr{E}(\mathbf{D}) \operatorname{rot} \mu_{0}^{-1 / 2}\left(\mathscr{L}_{J}^{0}+I_{J\left(\mu_{0}\right)}\right)^{-1} .
\end{aligned}
$$

Мы получили следующий результат.

Теорема 1.8. Пусть выполнены условия теоремь 1.1. Пусть $\mathscr{L}_{J, \varepsilon}=$ $\mu_{0}^{-1 / 2} \operatorname{rot}\left(\eta^{\varepsilon}\right)^{-1} \operatorname{rot} \mu_{0}^{-1 / 2}$ - часть оператора $\mathscr{L}_{\varepsilon}$, действующая в соленоидальном подпространстве (1.27). Пусть $\mathscr{L}_{J}^{0}=\mu_{0}^{-1 / 2} \operatorname{rot}\left(\eta^{0}\right)^{-1} \operatorname{rot} \mu_{0}^{-1 / 2}-$ часть эфбективного оператора $\mathscr{L}^{0}$, действующая в $J\left(\mu_{0}\right)$. Пусть $\mathscr{P}\left(\mu_{0}\right)$ - ортопроектор в $\mathfrak{G}$ на подпространство $J\left(\mu_{0}\right)$. Пусть корректор $K_{J}(\varepsilon)$ определен в $(1.31), \widetilde{K}_{1, J}(\varepsilon)$ определен в $(1.33)$ и $\widetilde{K}_{J}(\varepsilon)$ определен в (1.35). Тогда выполнены оценки (1.29), (1.30), (1.32) и (1.34). Постоянные $C_{1}^{\prime}$ из (1.29), $C_{2}^{\prime}$ из (1.30) u $C_{3}^{\prime}$ из (1.32) зависят лишь от $\left|\mu_{0}\right|,\left|\mu_{0}^{-1}\right|,\|\eta\|_{L_{\infty}},\left\|\eta^{-1}\right\|_{L_{\infty}}$ и от параметров решетки $\Gamma$, а постоянная $\mathscr{C}_{s}^{\prime}$ из (1.34) зависит от тех же величин, а такэе om $s$.

Применим теперь оценку (1.22) при $\mathbf{F} \in J\left(\mu_{0}\right)$. Тогда в силу распадения операторов $\mathscr{L}_{\varepsilon}$ и $\mathscr{L}^{0}$

$$
\mathbf{f}_{\varepsilon}=\left(\mathscr{L}_{J, \varepsilon}+I_{J\left(\mu_{0}\right)}\right)^{-1} \mathbf{F}, \quad \mathbf{f}_{0}=\left(\mathscr{L}_{J}^{0}+I_{J\left(\mu_{0}\right)}\right)^{-1} \mathbf{F}, \quad \mathbf{F} \in J\left(\mu_{0}\right) .
$$

Обозначая через $C_{5}^{\prime}$ постоянную $C_{5}$ при $\nu=1$, приходим к следующему результату.

Предложение 1.9. Пусть выполнены условия теоремы 1.5. Пусть векторфункции $\mathbf{f}_{\varepsilon} u \mathbf{f}_{0}$ определень в (1.36). Тогда при $0<\varepsilon \leqslant 1$ справедлива оценка

$$
\left\|\left(\eta^{\varepsilon}\right)^{-1} \operatorname{rot} \mu_{0}^{-1 / 2} \mathbf{f}_{\varepsilon}-\left(\mathbf{1}+Z^{\varepsilon}\right)\left(\eta^{0}\right)^{-1} \operatorname{rot} \mu_{0}^{-1 / 2} \mathbf{f}_{0}\right\|_{\mathfrak{G}} \leqslant C_{5}^{\prime} \varepsilon\|\mathbf{F}\|_{\mathfrak{G}},
$$

где $C_{5}^{\prime}$ зависит от $\left|\mu_{0}\right|,\left|\mu_{0}^{-1}\right|,\|\eta\|_{L_{\infty}},\left\|\eta^{-1}\right\|_{L_{\infty}}$ и от параметров решетки $\Gamma$.

Выделим особые случаи. Сужая операторы в $(1.23),(1.24)$ и $(1.25)$ на $J\left(\mu_{0}\right)$ и считая, что $\nu=1$, получаем следующий результат. 
Теорема 1.10. Пусть выполнены условия теоремы 1.6. Пусть оператор $\mathscr{L}_{J, \varepsilon}-$ часть оператора $\mathscr{L}_{\varepsilon}$ в подпространстве $J\left(\mu_{0}\right)$ и $\mathscr{L}_{J}^{0}-$ часть эффбективного оператора $\mathscr{L}^{0}$ в $J\left(\mu_{0}\right)$. Тогда при $0<\varepsilon \leqslant 1$ справедливы оченки

$$
\begin{aligned}
& \left\|\left(\mathscr{L}_{J, \varepsilon}+I_{J\left(\mu_{0}\right)}\right)^{-1}-\left(\mathscr{L}_{J}^{0}+I_{J\left(\mu_{0}\right)}\right)^{-1}\right\|_{J\left(\mu_{0}\right) \rightarrow J\left(\mu_{0}\right)} \leqslant \widetilde{C}_{2}^{\prime} \varepsilon^{2}, \\
& \left\|\left(\mathscr{L}_{J, \varepsilon}+I_{J\left(\mu_{0}\right)}\right)^{-1}-\left(\mathscr{L}_{J}^{0}+I_{J\left(\mu_{0}\right)}\right)^{-1}\right\|_{J\left(\mu_{0}\right) \rightarrow J^{1}\left(\mu_{0}\right)} \leqslant{\widetilde{C_{3}}}^{\prime} \varepsilon, \\
& \left\|\left(\mathscr{L}_{J, \varepsilon}+I_{J\left(\mu_{0}\right)}\right)^{-1}-\left(\mathscr{L}_{J}^{0}+I_{J\left(\mu_{0}\right)}\right)^{-1}\right\|_{J\left(\mu_{0}\right) \rightarrow J^{s}\left(\mu_{0}\right)} \leqslant \widetilde{\mathscr{C}}_{s}^{\prime} \varepsilon^{2-s}, \quad 0<s \leqslant 1 .
\end{aligned}
$$

Постояннье $\widetilde{C}_{2}^{\prime}, \widetilde{C}_{3}^{\prime}$ зависят лишь от $\left|\mu_{0}\right|,\left|\mu_{0}^{-1}\right|,\|\eta\|_{L_{\infty}},\left\|\eta^{-1}\right\|_{L_{\infty}}$ u oт nараметров решетки $\Gamma$, а постоянная $\widetilde{\mathscr{C}}_{s}^{\prime}$ зависит от тех же величин и от $s$.

Если выполнено условие $\eta^{0}=\bar{\eta}$, то из оценки (1.26) при $\mathbf{F} \in J\left(\mu_{0}\right)$ и при $\nu=1$ получаем следующий результат.

Предложение 1.11. Пусть выполнены условия теоремы 1.7. Пусть вектор-функиии $\mathbf{f}_{\varepsilon} u \mathbf{f}_{0}$ определены в (1.36). Тогда при $0<\varepsilon \leqslant 1$ справедлива оценка

$$
\left\|\left(\eta^{\varepsilon}\right)^{-1} \operatorname{rot} \mu_{0}^{-1 / 2} \mathbf{f}_{\varepsilon}-(\bar{\eta})^{-1} \operatorname{rot} \mu_{0}^{-1 / 2} \mathbf{f}_{0}\right\|_{\mathfrak{G}} \leqslant C_{5}^{\prime} \varepsilon\|\mathbf{F}\|_{\mathfrak{G}}
$$

где $C_{5}^{\prime}$ зависит от $\left|\mu_{0}\right|,\left|\mu_{0}^{-1}\right|,\|\eta\|_{L_{\infty}},\left\|\eta^{-1}\right\|_{L_{\infty}}$ и от параметров решетки $\Gamma$.

\section{§2. Стационарная система Максвелла}

2.1. Постановка задачи. Ниже $\mathbf{u}, \mathbf{v}$ - напряженности электрического и магнитного полей. Считаем, что магнитная проницаемость $\mu$ задается постоянной положительной матрицей $\mu_{0}$, а диэлектрическая проницаемость $\eta(\mathbf{x})$ есть Г-периодическая вещественная матрица-функция, удовлетворяющая условиям (1.2). Векторы электрической и магнитной индукций $\mathbf{w}, \mathbf{z}$ связаны с $\mathbf{u}, \mathbf{v}$ соотношениями $\mathbf{w}=\eta(\mathbf{x}) \mathbf{u}, \mathbf{z}=\mu_{0} \mathbf{v}$. Напомним обозначения: $\mathfrak{G}=L_{2}\left(\mathbb{R}^{3} ; \mathbb{C}^{3}\right)$, $J=\{\mathbf{f} \in \mathfrak{G}: \operatorname{div} \mathbf{f}=0\}$. Через $\mathfrak{G}\left(\eta^{-1}\right)=L_{2}\left(\mathbb{R}^{3} ; \mathbb{C}^{3} ; \eta^{-1}\right)$ обозначается «весовое» пространство со скалярным произведением $\left(\mathbf{f}_{1}, \mathbf{f}_{2}\right)_{\mathfrak{G}\left(\eta^{-1}\right)}=\left(\eta^{-1} \mathbf{f}_{1}, \mathbf{f}_{2}\right)_{\mathfrak{G}}$. Аналогично определяется $\mathfrak{G}\left(\mu_{0}^{-1}\right)$. Множество $J$ является замкнутым подпространством как в $\mathfrak{G}$, так и в весовых пространствах $\mathfrak{G}\left(\eta^{-1}\right)$ и $\mathfrak{G}\left(\mu_{0}^{-1}\right)$.

Оператор Максвелла $\mathscr{M}=\mathscr{M}\left(\eta, \mu_{0}\right)$, записанный в терминах индукций, действует в пространстве $J \oplus J$ и задается формулой

$$
\mathscr{M}=\mathscr{M}\left(\eta, \mu_{0}\right)=\left(\begin{array}{cc}
0 & i \operatorname{rot} \mu_{0}^{-1} \\
-i \operatorname{rot} \eta^{-1} & 0
\end{array}\right)
$$

на области определения

$$
\operatorname{Dom} \mathscr{M}\left(\eta, \mu_{0}\right)=\left\{(\mathbf{w}, \mathbf{z}): \mathbf{w} \in J, \mathbf{z} \in J, \operatorname{rot} \eta^{-1} \mathbf{w} \in \mathfrak{G}, \operatorname{rot} \mu_{0}^{-1} \mathbf{z} \in \mathfrak{G}\right\} .
$$

Оператор $\mathscr{M}$ замкнут, но не самосопряжен в $J \oplus J$ (относительно стандартного скалярного произведения в $\mathfrak{G} \oplus \mathfrak{G})$. Однако оператор $\mathscr{M}$ самосопряжен в пространстве $J \oplus J$, рассматриваемом как подпространство в $\mathfrak{G}\left(\eta^{-1}\right) \oplus \mathfrak{G}\left(\mu_{0}^{-1}\right)$.

Определим семейство операторов $\mathscr{M}_{\varepsilon}=\mathscr{M}\left(\eta^{\varepsilon}, \mu_{0}\right), \varepsilon>0$, действующих в $J \oplus J$. При этом Dom $\mathscr{M}_{\varepsilon}$ зависит от $\varepsilon$. Наша иель - изучение поведения резольвенты $\left(\mathscr{M}_{\varepsilon}-i I\right)^{-1}$ при $\varepsilon \rightarrow 0$. Рассмотрим уравнение

$$
\left(\mathscr{M}_{\varepsilon}-i I\right)\left(\begin{array}{c}
\mathbf{w}_{\varepsilon} \\
\mathbf{z}_{\varepsilon}
\end{array}\right)=\left(\begin{array}{c}
\mathbf{q} \\
\mathbf{r}
\end{array}\right), \quad \mathbf{q}, \mathbf{r} \in J
$$


Соответствующие напряженности заданы соотношениями $\mathbf{u}_{\varepsilon}=\left(\eta^{\varepsilon}\right)^{-1} \mathbf{w}_{\varepsilon}, \mathbf{v}_{\varepsilon}=$ $\mu_{0}^{-1} \mathbf{z}_{\varepsilon} \cdot$ В подробной записи (2.2) имеет вид

$$
\left.\begin{array}{r}
i \operatorname{rot} \mu_{0}^{-1} \mathbf{z}_{\varepsilon}-i \mathbf{w}_{\varepsilon}=\mathbf{q} \\
-i \operatorname{rot}\left(\eta^{\varepsilon}\right)^{-1} \mathbf{w}_{\varepsilon}-i \mathbf{z}_{\varepsilon}=\mathbf{r} \\
\operatorname{div} \mathbf{w}_{\varepsilon}=0, \quad \operatorname{div} \mathbf{z}_{\varepsilon}=0 .
\end{array}\right\}
$$

Представим решения в виде сумм (ср. [4, гл. 7], [10], [11]): $\mathbf{w}_{\varepsilon}=\mathbf{w}_{\varepsilon}^{(q)}+\mathbf{w}_{\varepsilon}^{(r)}$, $\mathbf{z}_{\varepsilon}=\mathbf{z}_{\varepsilon}^{(q)}+\mathbf{z}_{\varepsilon}^{(r)}$, где векторы $\mathbf{w}_{\varepsilon}^{(q)}, \mathbf{z}_{\varepsilon}^{(q)}$ являются решением системы (2.3) при $\mathbf{r}=0$, а векторы $\mathbf{w}_{\varepsilon}^{(r)}, \mathbf{z}_{\varepsilon}^{(r)}$ - решением системы $(2.3)$ при $\mathbf{q}=0$. Соответственно в виде сумм представляются и поля $\mathbf{u}_{\varepsilon}, \mathbf{v}_{\varepsilon}$.

2.2. Эффективный оператор. Пусть $\eta^{0}-$ эффективная матрица для оператора $-\operatorname{div} \eta \nabla$ (см. (1.5)) и $\mathscr{M}^{0}=\mathscr{M}\left(\eta^{0}, \mu_{0}\right)$ - эффективный оператор Максвелла. Введем в рассмотрение «усредненную» систему Максвелла

$$
\left(\mathscr{M}^{0}-i I\right)\left(\begin{array}{c}
\mathbf{w}_{0} \\
\mathbf{z}_{0}
\end{array}\right)=\left(\begin{array}{c}
\mathbf{q} \\
\mathbf{r}
\end{array}\right), \quad \mathbf{q}, \mathbf{r} \in J .
$$

Соответствующие напряженности $\mathbf{u}_{0}, \mathbf{v}_{0}$ связаны с $\mathbf{w}_{0}, \mathbf{z}_{0}$ соотношениями

$$
\mathbf{u}_{0}=\left(\eta^{0}\right)^{-1} \mathbf{w}_{0}, \quad \mathbf{v}_{0}=\mu_{0}^{-1} \mathbf{z}_{0} .
$$

Решения системы (2.4) мы также представим в виде суммы двух слагаемых: $\mathbf{w}_{0}=\mathbf{w}_{0}^{(q)}+\mathbf{w}_{0}^{(r)}, \mathbf{z}_{0}=\mathbf{z}_{0}^{(q)}+\mathbf{z}_{0}^{(r)}$, и аналогичное разложение имеет место для $\mathbf{u}_{0}, \mathbf{v}_{0}$.

Отметим, что симметрии между случаями $\mathbf{q}=0$ и $\mathbf{r}=0$ нет.

2.3. Случай $\mathbf{q}=0$. Аппроксимации полей $\mathbf{z}_{\varepsilon}^{(r)} \mathbf{u}_{\varepsilon}^{(r)}$. При $\mathbf{q}=0$ система (2.3) принимает вид

$$
\left.\begin{array}{r}
\mathbf{w}_{\varepsilon}^{(r)}=\operatorname{rot} \mu_{0}^{-1} \mathbf{z}_{\varepsilon}^{(r)}, \\
\operatorname{rot}\left(\eta^{\varepsilon}\right)^{-1} \mathbf{w}_{\varepsilon}^{(r)}+\mathbf{z}_{\varepsilon}^{(r)}=i \mathbf{r}, \\
\operatorname{div} \mathbf{w}_{\varepsilon}^{(r)}=0, \quad \operatorname{div} \mathbf{z}_{\varepsilon}^{(r)}=0 .
\end{array}\right\}
$$

Напряженности определяются формулами

$$
\mathbf{u}_{\varepsilon}^{(r)}=\left(\eta^{\varepsilon}\right)^{-1} \mathbf{w}_{\varepsilon}^{(r)}, \quad \mathbf{v}_{\varepsilon}^{(r)}=\mu_{0}^{-1} \mathbf{z}_{\varepsilon}^{(r)} .
$$

Из (2.6) следует, что $\mathbf{z}_{\varepsilon}^{(r)}$ является решением задачи

$$
\operatorname{rot}\left(\eta^{\varepsilon}\right)^{-1} \operatorname{rot} \mu_{0}^{-1} \mathbf{z}_{\varepsilon}^{(r)}+\mathbf{z}_{\varepsilon}^{(r)}=i \mathbf{r}, \quad \operatorname{div} \mathbf{z}_{\varepsilon}^{(r)}=0, \quad \mathbf{r} \in J
$$

Тогда функция $\mathbf{f}_{\varepsilon}^{(r)}=\mu_{0}^{-1 / 2} \mathbf{z}_{\varepsilon}^{(r)}$ является решением задачи

$\mu_{0}^{-1 / 2} \operatorname{rot}\left(\eta^{\varepsilon}\right)^{-1} \operatorname{rot} \mu_{0}^{-1 / 2} \mathbf{f}_{\varepsilon}^{(r)}+\mathbf{f}_{\varepsilon}^{(r)}=i \mu_{0}^{-1 / 2} \mathbf{r}, \quad \operatorname{div} \mu_{0}^{1 / 2} \mathbf{f}_{\varepsilon}^{(r)}=0, \quad \mu_{0}^{-1 / 2} \mathbf{r} \in J\left(\mu_{0}\right)$.

Следовательно,

$$
\mu_{0}^{-1 / 2} \mathbf{z}_{\varepsilon}^{(r)}=\left(\mathscr{L}_{J, \varepsilon}+I_{J\left(\mu_{0}\right)}\right)^{-1}\left(i \mu_{0}^{-1 / 2} \mathbf{r}\right) .
$$

Аналогично, для решений усредненной системы (2.4) при $\mathbf{q}=0$

$$
\mu_{0}^{-1 / 2} \mathbf{z}_{0}^{(r)}=\left(\mathscr{L}_{J}^{0}+I_{J\left(\mu_{0}\right)}\right)^{-1}\left(i \mu_{0}^{-1 / 2} \mathbf{r}\right)
$$


Из $(2.9),(2.10)$ и оценки $(1.29)$ получаем, что при $\varepsilon \rightarrow 0$ магнитные индукции $\mathbf{z}_{\varepsilon}^{(r)}$ сходятся к $\mathbf{z}_{0}^{(r)}$ по норме в $\mathfrak{G}$, причем

$$
\left\|\mathbf{z}_{\varepsilon}^{(r)}-\mathbf{z}_{0}^{(r)}\right\|_{\mathfrak{G}} \leqslant C_{1}^{\prime \prime} \varepsilon\|\mathbf{r}\|_{\mathfrak{G}}, \quad C_{1}^{\prime \prime}=C_{1}^{\prime}\left|\mu_{0}\right|^{1 / 2}\left|\mu_{0}^{-1}\right|^{1 / 2}, \quad 0<\varepsilon \leqslant 1 .
$$

Применяя (1.30), можно получить более точную аппроксимацию для $\mathbf{z}_{\varepsilon}^{(r)}$ в $\mathfrak{G}$ :

$$
\begin{gathered}
\left\|\mathbf{z}_{\varepsilon}^{(r)}-\mathbf{z}_{0}^{(r)}-\varepsilon \boldsymbol{\zeta}_{\varepsilon}^{(r)}\right\|_{\mathfrak{G}} \leqslant C_{2}^{\prime \prime} \varepsilon^{2}\|\mathbf{r}\|_{\mathfrak{G},} \quad C_{2}^{\prime \prime}=C_{2}^{\prime}\left|\mu_{0}\right|^{1 / 2}\left|\mu_{0}^{-1}\right|^{1 / 2}, \quad 0<\varepsilon \leqslant 1, \\
\boldsymbol{\zeta}_{\varepsilon}^{(r)}:=\mu_{0}^{1 / 2} K_{J}(\varepsilon)\left(i \mu_{0}^{-1 / 2} \mathbf{r}\right) .
\end{gathered}
$$

В силу (1.31) поле (2.13) разбивается на три слагаемых: $\boldsymbol{\zeta}_{\varepsilon}^{(r)}=\boldsymbol{\rho}_{\varepsilon}^{(r)}+\boldsymbol{\xi}_{\varepsilon}^{(r)}+\boldsymbol{\phi}_{0}^{(r)}$, где

$$
\begin{aligned}
& \boldsymbol{\rho}_{\varepsilon}^{(r)}=\mu_{0}^{1 / 2} \mathscr{P}\left(\mu_{0}\right) \mu_{0}^{-1 / 2} \Psi^{\varepsilon} \operatorname{rot}\left(\mu_{0}^{-1 / 2}\left(\mathscr{L}_{J}^{0}+I_{J\left(\mu_{0}\right)}\right)^{-1}\left(i \mu_{0}^{-1 / 2} \mathbf{r}\right)\right), \\
& \boldsymbol{\xi}_{\varepsilon}^{(r)}=\mu_{0}^{1 / 2}\left(\mathscr{L}_{J}^{0}+I_{J\left(\mu_{0}\right)}\right)^{-1} \mu_{0}^{-1 / 2} \operatorname{rot}\left(\left(\Psi^{\varepsilon}\right)^{t} \mu_{0}^{-1 / 2}\left(i \mu_{0}^{-1 / 2} \mathbf{r}\right)\right), \\
& \boldsymbol{\phi}_{0}^{(r)}=-\mu_{0}^{1 / 2}\left(\mathscr{L}_{J}^{0}+I_{J\left(\mu_{0}\right)}\right)^{-1} \mu_{0}^{-1 / 2} \mathscr{E}(\mathbf{D}) \operatorname{rot}\left(\mu_{0}^{-1 / 2}\left(\mathscr{L}_{J}^{0}+I_{J\left(\mu_{0}\right)}\right)^{-1}\left(i \mu_{0}^{-1 / 2} \mathbf{r}\right)\right) .
\end{aligned}
$$

Как отмечено в [11, п. 10.2],

$$
\mu_{0}^{1 / 2} \mathscr{P}\left(\mu_{0}\right) \mu_{0}^{-1 / 2}=\widetilde{\mathscr{P}}\left(\mu_{0}\right),
$$

где $\widetilde{\mathscr{P}}\left(\mu_{0}\right)$ - ортопроектор в весовом пространстве $\mathfrak{G}\left(\mu_{0}^{-1}\right)$ на подпространство $J$. Кроме того, из $(2.10)$ и из $(2.4)$ при $\mathbf{q}=0$ видно, что

$$
\operatorname{rot}\left(\mu_{0}^{-1 / 2}\left(\mathscr{L}_{J}^{0}+I_{J\left(\mu_{0}\right)}\right)^{-1}\left(i \mu_{0}^{-1 / 2} \mathbf{r}\right)\right)=\operatorname{rot} \mu_{0}^{-1} \mathbf{z}_{0}^{(r)}=\mathbf{w}_{0}^{(r)} .
$$

Из (2.14), (2.17), (2.18) следует, что $\boldsymbol{\rho}_{\varepsilon}^{(r)}=\widetilde{\mathscr{P}}\left(\mu_{0}\right) \Psi^{\varepsilon} \mathbf{w}_{0}^{(r)}$.

Отметим, далее, что поле (2.15), очевидно, является решением задачи

$$
\operatorname{rot}\left(\eta^{0}\right)^{-1} \operatorname{rot} \mu_{0}^{-1} \boldsymbol{\xi}_{\varepsilon}^{(r)}+\boldsymbol{\xi}_{\varepsilon}^{(r)}=i \operatorname{rot}\left(\Psi^{\varepsilon}\right)^{t} \mu_{0}^{-1} \mathbf{r}, \quad \operatorname{div} \boldsymbol{\xi}_{\varepsilon}^{(r)}=0 .
$$

Из (2.16) и (2.18) видно, что поле $\phi_{0}^{(r)}$ является решением задачи

$$
\operatorname{rot}\left(\eta^{0}\right)^{-1} \operatorname{rot} \mu_{0}^{-1} \phi_{0}^{(r)}+\phi_{0}^{(r)}=-\mathscr{E}(\mathbf{D}) \mathbf{w}_{0}^{(r)}, \quad \operatorname{div} \phi_{0}^{(r)}=0 .
$$

Получим теперь аппроксимацию индукции $\mathbf{z}_{\varepsilon}^{(r)}$ по $\left(\mathfrak{G}^{1}\right)$-норме. В силу $(1.32)$

$$
\left\|\mathbf{z}_{\varepsilon}^{(r)}-\mathbf{z}_{0}^{(r)}-\varepsilon \widetilde{\boldsymbol{\rho}}_{\varepsilon}^{(r)}\right\|_{\mathfrak{G}^{1}} \leqslant C_{3}^{\prime \prime} \varepsilon\|\mathbf{r}\|_{\mathfrak{G}}, \quad C_{3}^{\prime \prime}=C_{3}^{\prime}\left|\mu_{0}\right|^{1 / 2}\left|\mu_{0}^{-1}\right|^{1 / 2}, \quad 0<\varepsilon \leqslant 1,
$$

$$
\widetilde{\boldsymbol{\rho}}_{\varepsilon}^{(r)}:=\mu_{0}^{1 / 2} \widetilde{K}_{1, J}(\varepsilon)\left(i \mu_{0}^{-1 / 2} \mathbf{r}\right) .
$$

С учетом (1.33), (2.17) и (2.18) имеем $\widetilde{\boldsymbol{\rho}}_{\varepsilon}^{(r)}=\widetilde{\mathscr{P}}\left(\mu_{0}\right) \Psi^{\varepsilon} \Pi_{\varepsilon} \mathbf{w}_{0}^{(r)}$.

Наконец, оценка (1.34) вместе с (2.9), (2.10) позволяет получить аппроксимацию поля $\mathbf{z}_{\varepsilon}^{(r)}$ в классе $\mathfrak{G}^{s}, 0<s \leqslant 1$ :

$$
\left\|\mathbf{z}_{\varepsilon}^{(r)}-\mathbf{z}_{0}^{(r)}-\varepsilon \widetilde{\boldsymbol{\zeta}}_{\varepsilon}^{(r)}\right\|_{\mathfrak{G}^{s} s} \leqslant \mathscr{C}_{s}^{\prime \prime} \varepsilon^{2-s}\|\mathbf{r}\|_{\mathfrak{G}}, \quad \mathscr{C}_{s}^{\prime \prime}=\mathscr{C}_{s}^{\prime}\left|\mu_{0}\right|^{1 / 2}\left|\mu_{0}^{-1}\right|^{1 / 2}, \quad 0<\varepsilon \leqslant 1,
$$

где

$$
\widetilde{\boldsymbol{\zeta}}_{\varepsilon}^{(r)}=\mu_{0}^{1 / 2} \widetilde{K}_{J}(\varepsilon)\left(i \mu_{0}^{-1 / 2} \mathbf{r}\right)=\widetilde{\boldsymbol{\rho}}_{\varepsilon}^{(r)}+\Pi_{\varepsilon} \boldsymbol{\xi}_{\varepsilon}^{(r)}+\boldsymbol{\phi}_{0}^{(r)} .
$$


Мы учли здесь соотношения (1.35), (1.33), (2.22), (2.15), (2.16).

Аппроксимации для магнитной напряженности $\mathbf{v}_{\varepsilon}^{(r)}=\mu_{0}^{-1} \mathbf{z}_{\varepsilon}^{(r)}$ прямо вытекают из $(2.11),(2.12),(2.21)$ и $(2.23)$ с учетом соотношения $\mathbf{v}_{0}^{(r)}=\mu_{0}^{-1} \mathbf{z}_{0}^{(r)}$ (соответствующие оценки приведены ниже в (2.28), (2.31), (2.33), (2.35)).

2.4. Случай $q=0$. Аппроксимации полей $\mathbf{u}_{\varepsilon}^{(r)} \mathbf{u}_{\boldsymbol{\varepsilon}}^{(\boldsymbol{r})}$. В силу (2.6), (2.7) имеем

$$
\mathbf{u}_{\varepsilon}^{(r)}=\left(\eta^{\varepsilon}\right)^{-1} \mathbf{w}_{\varepsilon}^{(r)}=\left(\eta^{\varepsilon}\right)^{-1} \operatorname{rot} \mu_{0}^{-1} \mathbf{z}_{\varepsilon}^{(r)}=\left(\eta^{\varepsilon}\right)^{-1} \operatorname{rot} \mu_{0}^{-1 / 2} \mathbf{f}_{\varepsilon}^{(r)} .
$$

Отсюда видно, что $\mathbf{u}_{\varepsilon}^{(r)}$ играет роль «потока» относительно решения $\mathbf{f}_{\varepsilon}^{(r)}$ задачи (2.8). В силу предложения 1.9 при $0<\varepsilon \leqslant 1$ справедлива оценка

$\left\|\left(\eta^{\varepsilon}\right)^{-1} \operatorname{rot} \mu_{0}^{-1 / 2} \mathbf{f}_{\varepsilon}^{(r)}-\left(\mathbf{1}+Z^{\varepsilon}\right)\left(\eta^{0}\right)^{-1} \operatorname{rot} \mu_{0}^{-1 / 2} \mathbf{f}_{0}^{(r)}\right\|_{\mathfrak{G}} \leqslant C_{5}^{\prime \prime} \varepsilon\|\mathbf{r}\|_{\mathfrak{G}}, \quad C_{5}^{\prime \prime}=C_{5}^{\prime}\left|\mu_{0}^{-1}\right|^{1 / 2}$, где $\mathbf{f}_{0}^{(r)}=\mu_{0}^{-1 / 2} \mathbf{z}_{0}^{(r)}$. Поскольку $\left(\eta^{0}\right)^{-1} \operatorname{rot} \mu_{0}^{-1 / 2} \mathbf{f}_{0}^{(r)}=\left(\eta^{0}\right)^{-1} \operatorname{rot} \mu_{0}^{-1} \mathbf{z}_{0}^{(r)}=\mathbf{u}_{0}^{(r)}$, последняя оценка означает, что

$$
\left\|\mathbf{u}_{\varepsilon}^{(r)}-\left(\mathbf{1}+Z^{\varepsilon}\right) \mathbf{u}_{0}^{(r)}\right\|_{\mathfrak{G}} \leqslant C_{5}^{\prime \prime} \varepsilon\|\mathbf{r}\|_{\mathfrak{G},}, \quad 0<\varepsilon \leqslant 1
$$

Для электрической индукции $\mathbf{w}_{\varepsilon}^{(r)}=\eta^{\varepsilon} \mathbf{u}_{\varepsilon}^{(r)}$ из (2.25) получаем, что

$$
\left\|\mathbf{w}_{\varepsilon}^{(r)}-\widetilde{\eta}^{\varepsilon} \mathbf{u}_{0}^{(r)}\right\|_{\mathfrak{G}} \leqslant C_{5}^{\prime \prime}\|\eta\|_{L_{\infty}} \varepsilon\|\mathbf{r}\|_{\mathfrak{G},}, \quad 0<\varepsilon \leqslant 1
$$

где $\widetilde{\eta}(\mathbf{x})=\eta(\mathbf{x})(\mathbf{1}+Z(\mathbf{x}))$ - матрица со столбцами $\eta(\mathbf{x})\left(\nabla \Phi_{j}(\mathbf{x})+\mathbf{e}_{j}\right), j=1,2,3$. Введем матрицу $Y(\mathbf{x})=\widetilde{\eta}(\mathbf{x})\left(\eta^{0}\right)^{-1}-\mathbf{1}$ с нулевым средним значением. Тогда $\widetilde{\eta}^{\varepsilon} \mathbf{u}_{0}^{(r)}=\left(\mathbf{1}+Y^{\varepsilon}\right) \mathbf{w}_{0}^{(r)}$.

2.5. Случай $\mathbf{q}=\mathbf{0}$. Итоговый результат. Из сказанного в разд. 2.3, 2.4 непосредственно вытекает

Теорема 2.1. Пусть $\left(\mathbf{w}_{\varepsilon}^{(r)}, \mathbf{z}_{\varepsilon}^{(r)}\right)$ - решение системы (2.6), и пустъ поля $\mathbf{u}_{\varepsilon}^{(r)}, \mathbf{v}_{\varepsilon}^{(r)}$ определенъь в (2.7). Пусть $\left(\mathbf{w}_{0}^{(r)}, \mathbf{z}_{0}^{(r)}\right)$ - решение «усредненной»системь (2.4) при $\mathbf{q}=0$, и пусть $\mathbf{u}_{0}^{(r)}=\left(\eta^{0}\right)^{-1} \mathbf{w}_{0}^{(r)}, \mathbf{v}_{0}^{(r)}=\mu_{0}^{-1} \mathbf{z}_{0}^{(r)}$. Пyсmъ $Z(\mathbf{x})$ - матрица со столбиами $\nabla \Phi_{j}(\mathbf{x}), j=1,2,3$, где $\Phi_{j}$ есть Г-периодическое решение задачи (1.4) при $\mathbf{C}=\mathbf{e}_{j}$. Пусть $\widetilde{\eta}(\mathbf{x})$ - матрица со столбцами $\eta(\mathbf{x})\left(\nabla \Phi_{j}(\mathbf{x})+\mathbf{e}_{j}\right), j=1,2,3, u Y(\mathbf{x})=\widetilde{\eta}(\mathbf{x})\left(\eta^{0}\right)^{-1}-\mathbf{1}$. Тогда имеют место следующие утверждения:

$1^{\circ}$. Для всех четырех полей $\mathbf{u}_{\varepsilon}^{(r)}, \mathbf{w}_{\varepsilon}^{(r)}, \mathbf{v}_{\varepsilon}^{(r)}, \mathbf{z}_{\varepsilon}^{(r)}$ справедливы аппроксимации по норме в $\mathfrak{G}$ с погрешностью порядка $\varepsilon$ :

$$
\begin{aligned}
\left\|\mathbf{u}_{\varepsilon}^{(r)}-\left(\mathbf{1}+Z^{\varepsilon}\right) \mathbf{u}_{0}^{(r)}\right\|_{\mathfrak{G}} \leqslant C_{5}^{\prime \prime} \varepsilon\|\mathbf{r}\|_{\mathfrak{G},} & 0<\varepsilon \leqslant 1, \\
\left\|\mathbf{w}_{\varepsilon}^{(r)}-\left(\mathbf{1}+Y^{\varepsilon}\right) \mathbf{w}_{0}^{(r)}\right\|_{\mathfrak{G}} \leqslant C_{5}^{\prime \prime}\|\eta\|_{L_{\infty}} \varepsilon\|\mathbf{r}\|_{\mathfrak{G},} & 0<\varepsilon \leqslant 1, \\
\left\|\mathbf{v}_{\varepsilon}^{(r)}-\mathbf{v}_{0}^{(r)}\right\|_{\mathfrak{G}} \leqslant C_{1}^{\prime \prime}\left|\mu_{0}^{-1}\right| \varepsilon\|\mathbf{r}\|_{\mathfrak{G},} & 0<\varepsilon \leqslant 1, \\
\left\|\mathbf{z}_{\varepsilon}^{(r)}-\mathbf{z}_{0}^{(r)}\right\|_{\mathfrak{G}} \leqslant C_{1}^{\prime \prime} \varepsilon\|\mathbf{r}\|_{\mathfrak{G}}, & 0<\varepsilon \leqslant 1 .
\end{aligned}
$$


$2^{\circ}$. Для магнитной индукиии $\mathbf{z}_{\varepsilon}^{(r)}$ и магнитной напряженности $\mathbf{v}_{\varepsilon}^{(r)}$ справедливы следующие аппроксимации по норме в $\mathfrak{G}$ с погрешностью порлдка $\varepsilon^{2}$ :

$$
\begin{array}{cl}
\left\|\mathbf{z}_{\varepsilon}^{(r)}-\mathbf{z}_{0}^{(r)}-\varepsilon \boldsymbol{\zeta}_{\varepsilon}^{(r)}\right\|_{\mathfrak{G}} \leqslant C_{2}^{\prime \prime} \varepsilon^{2}\|\mathbf{r}\|_{\mathfrak{G},} & 0<\varepsilon \leqslant 1, \\
\left\|\mathbf{v}_{\varepsilon}^{(r)}-\mathbf{v}_{0}^{(r)}-\varepsilon \mu_{0}^{-1} \boldsymbol{\zeta}_{\varepsilon}^{(r)}\right\|_{\mathfrak{G}} \leqslant C_{2}^{\prime \prime}\left|\mu_{0}^{-1}\right| \varepsilon^{2}\|\mathbf{r}\|_{\mathfrak{G},} & 0<\varepsilon \leqslant 1 .
\end{array}
$$

Здесь поправка $\boldsymbol{\zeta}_{\varepsilon}^{(r)}$ определяется следующим образом. Пусть $\Psi(\mathbf{x})$ - матрица со столбиами $\operatorname{rot} \mathbf{p}_{j}, j=1,2,3$, где $\mathbf{p}_{j}$ есть Г-периодическое решение задачи (1.9). Пусть $\widetilde{\mathscr{P}}\left(\mu_{0}\right)$ - ортопроектор в весовом пространстве $\mathfrak{G}\left(\mu_{0}^{-1}\right)$ на соленоидальное подпространство $Ј$. Пусть $\boldsymbol{\xi}_{\varepsilon}^{(r)}$ - решение задачи (2.19) и $\phi_{0}^{(r)}$ - решение задачи (2.20), где ДО $\mathscr{E}(\mathbf{D})$ определен в (1.10), (1.11). Тогда $\boldsymbol{\zeta}_{\varepsilon}^{(r)}=\widetilde{\mathscr{P}}\left(\mu_{0}\right) \Psi^{\varepsilon} \mathbf{w}_{0}^{(r)}+\boldsymbol{\xi}_{\varepsilon}^{(r)}+\phi_{0}^{(r)}$.

$3^{\circ}$. Для полей $\mathbf{z}_{\varepsilon}^{(r)} u \mathbf{v}_{\varepsilon}^{(r)}$ справедливы следующие аппроксимации по норме 8 $\mathfrak{G}^{1}$ с погрешностью порядка $\varepsilon$ :

$$
\begin{array}{cl}
\left\|\mathbf{z}_{\varepsilon}^{(r)}-\mathbf{z}_{0}^{(r)}-\varepsilon \widetilde{\boldsymbol{\rho}}_{\varepsilon}^{(r)}\right\|_{\mathfrak{G}^{1} 1} \leqslant C_{3}^{\prime \prime} \varepsilon\|\mathbf{r}\|_{\mathfrak{G},}, & 0<\varepsilon \leqslant 1, \\
\left\|\mathbf{v}_{\varepsilon}^{(r)}-\mathbf{v}_{0}^{(r)}-\varepsilon \mu_{0}^{-1} \widetilde{\boldsymbol{\rho}}_{\varepsilon}^{(r)}\right\|_{\mathfrak{G}^{1}} \leqslant C_{3}^{\prime \prime}\left|\mu_{0}^{-1}\right| \varepsilon\|\mathbf{r}\|_{\mathfrak{G}}, & 0<\varepsilon \leqslant 1 .
\end{array}
$$

Здесь $\widetilde{\boldsymbol{\rho}}_{\varepsilon}^{(r)}=\widetilde{\mathscr{P}}\left(\mu_{0}\right) \Psi^{\varepsilon} \Pi_{\varepsilon} \mathbf{w}_{0}^{(r)}$, а $\Pi_{\varepsilon}$ есть ПДО в $\mathfrak{G}$ с символом $\chi_{\widetilde{\Omega} / \varepsilon}$.

$4^{\circ}$. Для полей $\mathbf{z}_{\varepsilon}^{(r)} u \mathbf{v}_{\varepsilon}^{(r)}$ справедливы следующие аппроксимации по норме в $\mathfrak{G}^{s}$ при $0<s \leqslant 1$ с погрешностью порядка $\varepsilon^{2-s}$ :

$$
\begin{array}{cc}
\left\|\mathbf{z}_{\varepsilon}^{(r)}-\mathbf{z}_{0}^{(r)}-\varepsilon \widetilde{\boldsymbol{\zeta}}_{\varepsilon}^{(r)}\right\|_{\mathfrak{G}^{s}} \leqslant \mathscr{C}_{s}^{\prime \prime} \varepsilon^{2-s}\|\mathbf{r}\|_{\mathfrak{G},}, & 0<\varepsilon \leqslant 1, \\
\left\|\mathbf{v}_{\varepsilon}^{(r)}-\mathbf{v}_{0}^{(r)}-\varepsilon \mu_{0}^{-1} \widetilde{\boldsymbol{\zeta}}_{\varepsilon}^{(r)}\right\|_{\mathfrak{G}^{s}} \leqslant \mathscr{C}_{s}^{\prime \prime}\left|\mu_{0}^{-1}\right| \varepsilon^{2-s}\|\mathbf{r}\|_{\mathfrak{G},}, & 0<\varepsilon \leqslant 1 .
\end{array}
$$

Здесъ $\widetilde{\boldsymbol{\zeta}}_{\varepsilon}^{(r)}=\widetilde{\boldsymbol{\rho}}_{\varepsilon}^{(r)}+\Pi_{\varepsilon} \boldsymbol{\xi}_{\varepsilon}^{(r)}+\boldsymbol{\phi}_{0}^{(r)}$.

Постоянные в оченках (2.26)-(2.33) зависят лишь от $\left|\mu_{0}\right|,\left|\mu_{0}^{-1}\right|,\|\eta\|_{L_{\infty}}$, $\left\|\eta^{-1}\right\|_{L_{\infty}}$ и от параметров решетки $\Gamma$, а постолнная $\mathscr{C}_{s}^{\prime \prime}$ из $(2.34),(2.35)$ зависит от тех же величин и от $s$.

Отметим, что при $\varepsilon \rightarrow 0$ функции $\mathbf{u}_{\varepsilon}^{(r)}$ слабо сходятся в $\mathfrak{G}$ к $\mathbf{u}_{0}^{(r)}$, а $\mathbf{w}_{\varepsilon}^{(r)}$ слабо сходятся в $\mathfrak{G}$ к $\mathbf{w}_{0}^{(r)}$. Это согласуется с оценками (2.26), (2.27): поправочные члены $Z^{\varepsilon} \mathbf{u}_{0}^{(r)}, Y^{\varepsilon} \mathbf{w}_{0}^{(r)}$ слабо сходятся в $\mathfrak{G}$ к нулю в силу свойства среднего значения $(Z(\mathbf{x})$ и $Y(\mathbf{x})$ - периодические матрицы-функции с нулевым средним значением).

2.6. Частные случаи при $\mathbf{q}=\mathbf{0}$. Если $\eta^{0}=\underline{\eta}$ (т. е. столбцы матрицы $\eta^{-1}$ потенциальны), то применима теорема 1.10. Вместе с соотношениями (2.9), (2.10) эта теорема дает аппроксимации для полей $\mathbf{v}_{\varepsilon}^{(r)}, \mathbf{z}_{\varepsilon}^{(r)}$, причем поправки (корректоры) обращаются в нуль. Кроме того, при $\eta^{0}=\underline{\eta}$ выполнено соотношение $\widetilde{\eta}(\mathbf{x})=\eta^{0}=\underline{\eta}$ (ср. [6, замечание 3.5]). Поэтому $Y(\overline{\mathbf{x}})=0$, а $\mathbf{1}+Z(\mathbf{x})=$ $\eta(\mathbf{x})^{-1} \eta^{0}$, и аппроксимации $(2.27),(2.26)$ упрощаются. Суммируя сказанное, получаем следующую теорему, в которой мы используем обозначения

$$
\widetilde{C}_{2}^{\prime \prime}=\widetilde{C}_{2}^{\prime}\left|\mu_{0}\right|^{1 / 2}\left|\mu_{0}^{-1}\right|^{1 / 2}, \quad \widetilde{C}_{3}^{\prime \prime}=\widetilde{C}_{3}^{\prime}\left|\mu_{0}\right|^{1 / 2}\left|\mu_{0}^{-1}\right|^{1 / 2}, \quad \widetilde{\mathscr{C}}_{s}^{\prime \prime}=\widetilde{\mathscr{C}}_{s}^{\prime}\left|\mu_{0}\right|^{1 / 2}\left|\mu_{0}^{-1}\right|^{1 / 2} .
$$


Теорема 2.2. Пусть выполнены условия теоремы 2.1. Пусть, кроме того, выполнено соотношение $\eta^{0}=\underline{\eta}$. Тогда для магнитной индукиии $\mathbf{z}_{\varepsilon}^{(r)}$ и магнитной напряюсенности $\mathbf{v}_{\varepsilon}^{(r)}$ справедливъ следующие аппроксимащии:

$$
\begin{array}{ll}
\left\|\mathbf{z}_{\varepsilon}^{(r)}-\mathbf{z}_{0}^{(r)}\right\|_{\mathfrak{G}} \leqslant \widetilde{C}_{2}^{\prime \prime} \varepsilon^{2}\|\mathbf{r}\|_{\mathfrak{G},}, & 0<\varepsilon \leqslant 1, \\
\left\|\mathbf{v}_{\varepsilon}^{(r)}-\mathbf{v}_{0}^{(r)}\right\|_{\mathfrak{G}} \leqslant \widetilde{C}_{2}^{\prime \prime}\left|\mu_{0}^{-1}\right| \varepsilon^{2}\|\mathbf{r}\|_{\mathfrak{G},}, & 0<\varepsilon \leqslant 1, \\
\left\|\mathbf{z}_{\varepsilon}^{(r)}-\mathbf{z}_{0}^{(r)}\right\|_{\mathfrak{G}^{1}} \leqslant \widetilde{C}_{3}^{\prime \prime} \varepsilon\|\mathbf{r}\|_{\mathfrak{G},}, & 0<\varepsilon \leqslant 1, \\
\left\|\mathbf{v}_{\varepsilon}^{(r)}-\mathbf{v}_{0}^{(r)}\right\|_{\mathfrak{G}^{1}} \leqslant \widetilde{C}_{3}^{\prime \prime}\left|\mu_{0}^{-1}\right| \varepsilon\left\|_{\mathbf{r}}\right\|_{\mathfrak{G},} & 0<\varepsilon \leqslant 1, \\
\left\|\mathbf{z}_{\varepsilon}^{(r)}-\mathbf{z}_{0}^{(r)}\right\|_{\mathfrak{G}^{s}} \leqslant \widetilde{\mathscr{C}}_{s}^{\prime \prime} \varepsilon^{2-s}\|\mathbf{r}\|_{\mathfrak{G},}, 0<s \leqslant 1,0<\varepsilon \leqslant 1, \\
\left\|\mathbf{v}_{\varepsilon}^{(r)}-\mathbf{v}_{0}^{(r)}\right\|_{\mathfrak{G}^{s}} \leqslant \widetilde{\mathscr{C}}_{s}^{\prime \prime}\left|\mu_{0}^{-1}\right| \varepsilon^{2-s}\|\mathbf{r}\|_{\mathfrak{G}}, & 0<s \leqslant 1,0<\varepsilon \leqslant 1 .
\end{array}
$$

Для электрических индукиии $\mathbf{w}_{\varepsilon}^{(r)}$ и напряюенности $\mathbf{u}_{\varepsilon}^{(r)}$

$$
\begin{array}{ll}
\left\|\mathbf{w}_{\varepsilon}^{(r)}-\mathbf{w}_{0}^{(r)}\right\|_{\mathfrak{G}} \leqslant C_{5}^{\prime \prime}\|\eta\|_{L_{\infty}} \varepsilon\|\mathbf{r}\|_{\mathfrak{G},}, & 0<\varepsilon \leqslant 1, \\
\left\|\mathbf{u}_{\varepsilon}^{(r)}-\left(\eta^{\varepsilon}\right)^{-1} \mathbf{w}_{0}^{(r)}\right\|_{\mathfrak{G}} \leqslant C_{5}^{\prime \prime} \varepsilon\|\mathbf{r}\|_{\mathfrak{G},}, & 0<\varepsilon \leqslant 1 .
\end{array}
$$

Постояннье $\widetilde{C}_{2}^{\prime \prime}, \widetilde{C}_{3}^{\prime \prime}, C_{5}^{\prime \prime}$ зависят лишь от $\left|\mu_{0}\right|,\left|\mu_{0}^{-1}\right|,\|\eta\|_{L_{\infty}},\left\|\eta^{-1}\right\|_{L_{\infty}} u$ om параметров решетки $\Gamma$, a $\widetilde{\mathscr{C}}_{s}^{\prime \prime}$ зависит от тех же величин, а также от $s$.

Пусть теперь выполнено соотношение $\eta^{0}=\bar{\eta}$, т. е. столбцы матрицы $\eta(\mathbf{x})$ соленоидальны. Тогда применимо предложение 1.11. С учетом (2.24) приходим к следующему результату.

Теорема 2.3. Пусть выполнень условия теоремы 2.1. Кроме того, пусть $\eta^{0}=\bar{\eta}$. Тогда для электрической напряэенности $\mathbf{u}_{\varepsilon}^{(r)}$ справедлива аппроксимаиця

$$
\left\|\mathbf{u}_{\varepsilon}^{(r)}-\mathbf{u}_{0}^{(r)}\right\|_{\mathfrak{G}} \leqslant C_{5}^{\prime \prime} \varepsilon\|\mathbf{r}\|_{\mathfrak{G},}, \quad 0<\varepsilon \leqslant 1,
$$

а для электрической индукции $\mathbf{w}_{\varepsilon}^{(r)}-$ аппроксимаиия

$$
\left\|\mathbf{w}_{\varepsilon}^{(r)}-\eta^{\varepsilon} \mathbf{u}_{0}^{(r)}\right\|_{\mathfrak{G}} \leqslant C_{5}^{\prime \prime}\|\eta\|_{L_{\infty}} \varepsilon\|\mathbf{r}\|_{\mathfrak{G},}, \quad 0<\varepsilon \leqslant 1 .
$$

Постоянная $C_{5}^{\prime \prime}$ зависит лишь от $\left|\mu_{0}\right|,\left|\mu_{0}^{-1}\right|,\|\eta\|_{L_{\infty}},\left\|\eta^{-1}\right\|_{L_{\infty}}$ u от параметров решетки Г.

2.7. Случай $\mathbf{r}=\mathbf{0}$. Аппроксимация электрических полей. При $\mathbf{r}=0$ система (2.3) для индукций $\mathbf{w}_{\varepsilon}^{(q)}$ и $\mathbf{z}_{\varepsilon}^{(q)}$ имеет вид

$$
\left.\begin{array}{r}
\mathbf{z}_{\varepsilon}^{(q)}=-\operatorname{rot}\left(\eta^{\varepsilon}\right)^{-1} \mathbf{w}_{\varepsilon}^{(q)}, \\
-\operatorname{rot}\left(\mu_{0}\right)^{-1} \mathbf{z}_{\varepsilon}^{(q)}+\mathbf{w}_{\varepsilon}^{(q)}=i \mathbf{q}, \\
\operatorname{div} \mathbf{w}_{\varepsilon}^{(q)}=0, \quad \operatorname{div} \mathbf{z}_{\varepsilon}^{(q)}=0 .
\end{array}\right\}
$$

Напряженности записываются в виде

$$
\mathbf{u}_{\varepsilon}^{(q)}=\left(\eta^{\varepsilon}\right)^{-1} \mathbf{w}_{\varepsilon}^{(q)}, \quad \mathbf{v}_{\varepsilon}^{(q)}=\mu_{0}^{-1} \mathbf{z}_{\varepsilon}^{(q)} .
$$

В [10], [11] были получены аппроксимации в пространстве $\mathfrak{G}$ для полей $\mathbf{w}_{\varepsilon}^{(q)}$ и $\mathbf{u}_{\varepsilon}^{(q)}$ с погрешностью порядка $\varepsilon$. При этом обе проницаемости $\eta, \mu$ считалисъ 
Г-периодическими. Сформулируем соответствующий результат применительно к случаю $\mu=\mu_{0}=$ Const. Пусть $\left(\mathbf{w}_{0}^{(q)}, \mathbf{z}_{0}^{(q)}\right)$ - решение усредненной системы (2.4) при $\mathbf{r}=0$. Положим $\mathbf{u}_{0}^{(q)}=\left(\eta^{0}\right)^{-1} \mathbf{w}_{0}^{(q)}, \mathbf{v}_{0}^{(q)}=\left(\mu_{0}\right)^{-1} \mathbf{z}_{0}^{(q)}$. Пусть $\left(\widehat{\mathbf{w}}_{\varepsilon}^{(q)}, \widehat{\mathbf{z}}_{\varepsilon}^{(q)}\right)$ - решение «поправочной» системы

$$
\left(\mathscr{M}^{0}-i I\right)\left(\begin{array}{c}
\widehat{\mathbf{w}}_{\varepsilon}^{(q)} \\
\widehat{\mathbf{z}}_{\varepsilon}^{(q)}
\end{array}\right)=\left(\begin{array}{c}
\mathbf{q}_{\varepsilon} \\
0
\end{array}\right),
$$

где $\mathbf{q}_{\varepsilon}=\widetilde{\mathscr{P}}\left(\eta^{0}\right)\left(Z^{\varepsilon}\right)^{t} \mathbf{q}$. Здесь $\widetilde{\mathscr{P}}\left(\eta^{0}\right)-$ ортопроектор в весовом пространстве $\mathfrak{G}\left(\left(\eta^{0}\right)^{-1}\right)$ на подпространство $J$. Матрица $Z(\mathbf{x})$ - та же, что и выше. Пусть

$$
\widehat{\mathbf{u}}_{\varepsilon}^{(q)}=\left(\eta^{0}\right)^{-1} \widehat{\mathbf{w}}_{\varepsilon}^{(q)}, \quad \widehat{\mathbf{v}}_{\varepsilon}^{(q)}=\mu_{0}^{-1} \widehat{\mathbf{z}}_{\varepsilon}^{(q)} .
$$

Положим

$$
\begin{gathered}
\widetilde{\mathbf{u}}_{\varepsilon}^{(q)}=\left(\mathbf{1}+Z^{\varepsilon}\right)\left(\mathbf{u}_{0}^{(q)}+\widehat{\mathbf{u}}_{\varepsilon}^{(q)}\right), \\
\widetilde{\mathbf{w}}_{\varepsilon}^{(q)}=\widetilde{\eta}^{\varepsilon}\left(\mathbf{u}_{0}^{(q)}+\widehat{\mathbf{u}}_{\varepsilon}^{(q)}\right)=\left(\mathbf{1}+Y^{\varepsilon}\right)\left(\mathbf{w}_{0}^{(q)}+\widehat{\mathbf{w}}_{\varepsilon}^{(q)}\right) .
\end{gathered}
$$

Из [11, теорема 12.2] прямо вытекают аппроксимации для полей $\mathbf{u}_{\varepsilon}^{(q)}, \mathbf{w}_{\varepsilon}^{(q)}$ :

$$
\left\|\mathbf{u}_{\varepsilon}^{(q)}-\widetilde{\mathbf{u}}_{\varepsilon}^{(q)}\right\|_{\mathfrak{G}} \leqslant C_{6} \varepsilon\|\mathbf{q}\|_{\mathfrak{G}}, \quad\left\|\mathbf{w}_{\varepsilon}^{(q)}-\widetilde{\mathbf{w}}_{\varepsilon}^{(q)}\right\|_{\mathfrak{G}} \leqslant C_{7} \varepsilon\|\mathbf{q}\|_{\mathfrak{G}}, \quad 0<\varepsilon \leqslant 1 .
$$

Постоянные $C_{6}, C_{7}$ зависят лишь от $\left|\mu_{0}\right|,\left|\mu_{0}^{-1}\right|,\|\eta\|_{L_{\infty}},\left\|\eta^{-1}\right\|_{L_{\infty}}$ и от параметров решетки $Г$.

2.8. Случай $\mathbf{r}=0$. Аппроксимация магнитных полей. Для аппроксимации в $\mathfrak{G}$ полей $\mathbf{z}_{\varepsilon}^{(q)}$ и $\mathbf{v}_{\varepsilon}^{(q)}$ нам приходится использовать специфику случая $\mu=\mu_{0}$. Из (2.38) видно, что $\mathbf{z}_{\varepsilon}^{(q)}$ является решением задачи

$$
\operatorname{rot}\left(\eta^{\varepsilon}\right)^{-1} \operatorname{rot} \mu_{0}^{-1} \mathbf{z}_{\varepsilon}^{(q)}+\mathbf{z}_{\varepsilon}^{(q)}=-i \operatorname{rot}\left(\eta^{\varepsilon}\right)^{-1} \mathbf{q}, \quad \operatorname{div} \mathbf{z}_{\varepsilon}^{(q)}=0 .
$$

Тогда функция $\mathbf{f}_{\varepsilon}^{(q)}=\mu_{0}^{-1 / 2} \mathbf{z}_{\varepsilon}^{(q)}$ является решением задачи

$$
\mu_{0}^{-1 / 2} \operatorname{rot}\left(\eta^{\varepsilon}\right)^{-1} \operatorname{rot} \mu_{0}^{-1 / 2} \mathbf{f}_{\varepsilon}^{(q)}+\mathbf{f}_{\varepsilon}^{(q)}=-i \mu_{0}^{-1 / 2} \operatorname{rot}\left(\eta^{\varepsilon}\right)^{-1} \mathbf{q}, \quad \operatorname{div} \mu_{0}^{1 / 2} \mathbf{f}_{\varepsilon}^{(q)}=0 .
$$

Следовательно,

$$
\mu_{0}^{-1 / 2} \mathbf{z}_{\varepsilon}^{(q)}=\mathbf{f}_{\varepsilon}^{(q)}=-\left(\mathscr{L}_{J, \varepsilon}+I_{J\left(\mu_{0}\right)}\right)^{-1}\left(i \mu_{0}^{-1 / 2} \operatorname{rot}\left(\eta^{\varepsilon}\right)^{-1} \mathbf{q}\right) .
$$

Воспользуемся оценкой (1.37), которую запишем в операторном виде:

$$
\begin{aligned}
\|\left(\eta^{\varepsilon}\right)^{-1} & \operatorname{rot} \mu_{0}^{-1 / 2}\left(\mathscr{L}_{J, \varepsilon}+I_{J\left(\mu^{0}\right)}\right)^{-1} \\
& -\left(\mathbf{1}+Z^{\varepsilon}\right)\left(\eta^{0}\right)^{-1} \operatorname{rot} \mu_{0}^{-1 / 2}\left(\mathscr{L}_{J}^{0}+I_{J\left(\mu^{0}\right)}\right)^{-1} \|_{J\left(\mu_{0}\right) \rightarrow \mathfrak{G}} \leqslant C_{5}^{\prime} \varepsilon,
\end{aligned}
$$

где $0<\varepsilon \leqslant 1$. Переходя к сопряженным операторам, при $0<\varepsilon \leqslant 1$ получаем

$$
\begin{aligned}
\|\left(\mathscr{L}_{J, \varepsilon}+I_{J\left(\mu^{0}\right)}\right)^{-1} \mu_{0}^{-1 / 2} \operatorname{rot}\left(\eta^{\varepsilon}\right)^{-1} & \\
& -\left(\mathscr{L}_{J}^{0}+I_{J\left(\mu^{0}\right)}\right)^{-1} \mu_{0}^{-1 / 2} \operatorname{rot}\left(\eta^{0}\right)^{-1}\left(\mathbf{1}+\left(Z^{\varepsilon}\right)^{t}\right) \|_{\mathfrak{G} \rightarrow J\left(\mu_{0}\right)} \leqslant C_{5}^{\prime} \varepsilon .
\end{aligned}
$$

Отсюда и из (2.42) следует, что

$$
\left\|\mathbf{z}_{\varepsilon}^{(q)}-\widetilde{\mathbf{z}}_{\varepsilon}^{(q)}\right\|_{\mathfrak{G}} \leqslant C_{5}^{\prime}\left|\mu_{0}\right|^{1 / 2} \varepsilon\|\mathbf{q}\|_{\mathfrak{G}}, \quad 0<\varepsilon \leqslant 1,
$$

где

$$
\widetilde{\mathbf{z}}_{\varepsilon}^{(q)}=-\mu_{0}^{1 / 2}\left(\mathscr{L}_{J}^{0}+I_{J\left(\mu^{0}\right)}\right)^{-1} \mu_{0}^{-1 / 2} \operatorname{rot}\left(\eta^{0}\right)^{-1}\left(\mathbf{1}+\left(Z^{\varepsilon}\right)^{t}\right) i \mathbf{q} .
$$


Нетрудно видеть, что $\widetilde{\mathbf{z}}_{\varepsilon}^{(q)}=\mathbf{z}_{0}^{(q)}+\widehat{\mathbf{z}}_{\varepsilon}^{(q)}$, где $\widehat{\mathbf{z}}_{\varepsilon}^{(q)}$ является решением задачи

$$
\operatorname{rot}\left(\eta^{0}\right)^{-1} \operatorname{rot} \mu_{0}^{-1} \widehat{\mathbf{z}}_{\varepsilon}^{(q)}+\widehat{\mathbf{z}}_{\varepsilon}^{(q)}=-i \operatorname{rot}\left(\eta^{0}\right)^{-1}\left(Z^{\varepsilon}\right)^{t} \mathbf{q}, \quad \operatorname{div} \widehat{\mathbf{z}}_{\varepsilon}^{(q)}=0 .
$$

Сопоставляя (2.44) и (2.40), убеждаемся, что $\widehat{\mathbf{z}}_{\varepsilon}^{(q)}$ (вместе с $\widehat{\mathbf{w}}_{\varepsilon}^{(q)}=i \mathbf{q}_{\varepsilon}+$ $\left.\operatorname{rot} \mu_{0}^{-1} \widehat{\mathbf{z}}_{\varepsilon}^{(q)}\right)$ есть решение «поправочной» задачи $(2.40)$.

Далее, из $(2.39),(2.41)$ и (2.43) получаем

$$
\left\|\mathbf{v}_{\varepsilon}^{(q)}-\widetilde{\mathbf{v}}_{\varepsilon}^{(q)}\right\|_{\mathfrak{G}} \leqslant C_{5}^{\prime}\left|\mu_{0}^{-1}\left\|\left.\mu_{0}\right|^{1 / 2} \varepsilon\right\| \mathbf{q} \|_{\mathfrak{G},}, \quad 0<\varepsilon \leqslant 1\right.
$$

где $\widetilde{\mathbf{v}}_{\varepsilon}^{(q)}=\mathbf{v}_{0}^{(q)}+\widehat{\mathbf{v}}_{\varepsilon}^{(q)}$.

\section{9. Итоговый результат при $\mathbf{r}=\mathbf{0}$ дает следующая}

Теорема 2.4. Пусть $\left(\mathbf{w}_{\varepsilon}^{(q)}, \mathbf{z}_{\varepsilon}^{(q)}\right)$ - решение системъ (2.38), и пусть по$л я \mathbf{u}_{\varepsilon}^{(q)}, \mathbf{v}_{\varepsilon}^{(q)}$ определенъ в (2.39). Пусть $\left(\mathbf{w}_{0}^{(q)}, \mathbf{z}_{0}^{(q)}\right)$ - решение «усредненной» системы (2.4) при $\mathbf{r}=0$, и пусть $\mathbf{u}_{0}^{(q)}=\left(\eta^{0}\right)^{-1} \mathbf{w}_{0}^{(q)}, \mathbf{v}_{0}^{(q)}=\mu_{0}^{-1} \mathbf{z}_{0}^{(q)}$. Пyсmъ $\left(\widehat{\mathbf{w}}_{\varepsilon}^{(q)}, \widehat{\mathbf{z}}_{\varepsilon}^{(q)}\right)$ - решение «поправочной» системы $(2.40)$, а поля $\left(\widehat{\mathbf{u}}_{\varepsilon}^{(q)}, \widehat{\mathbf{v}}_{\varepsilon}^{(q)}\right)$ определены в (2.41). Пусть $Z(\mathbf{x}), Y(\mathbf{x})$ - матрицы-функиии, определеннье в условиях теоремы 2.1. Тогда для всех четырех полей $\mathbf{u}_{\varepsilon}^{(q)}, \mathbf{w}_{\varepsilon}^{(q)}, \mathbf{v}_{\varepsilon}^{(q)}, \mathbf{z}_{\varepsilon}^{(q)}$ справедливъ следующие аппроксимащии по норме в $\mathfrak{G}$ с погрешностью порядка $\varepsilon$ :

$$
\begin{array}{cl}
\left\|\mathbf{u}_{\varepsilon}^{(q)}-\widetilde{\mathbf{u}}_{\varepsilon}^{(q)}\right\|_{\mathfrak{G}} \leqslant C_{6} \varepsilon\|\mathbf{q}\|_{\mathfrak{G}}, & 0<\varepsilon \leqslant 1, \\
\left\|\mathbf{w}_{\varepsilon}^{(q)}-\widetilde{\mathbf{w}}_{\varepsilon}^{(q)}\right\|_{\mathfrak{G}} \leqslant C_{7} \varepsilon\|\mathbf{q}\|_{\mathfrak{G},}, & 0<\varepsilon \leqslant 1, \\
\left\|\mathbf{v}_{\varepsilon}^{(q)}-\widetilde{\mathbf{v}}_{\varepsilon}^{(q)}\right\|_{\mathfrak{G}} \leqslant C_{5}^{\prime}\left|\mu_{0}^{-1}\left\|\left.\mu_{0}\right|^{1 / 2} \varepsilon\right\| \mathbf{q} \|_{\mathfrak{G},},\right. & 0<\varepsilon \leqslant 1, \\
\left\|\mathbf{z}_{\varepsilon}^{(q)}-\widetilde{\mathbf{z}}_{\varepsilon}^{(q)}\right\|_{\mathfrak{G}} \leqslant C_{5}^{\prime}\left|\mu_{0}\right|^{1 / 2} \varepsilon\|\mathbf{q}\|_{\mathfrak{G},} & 0<\varepsilon \leqslant 1,
\end{array}
$$

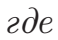

$$
\begin{gathered}
\widetilde{\mathbf{u}}_{\varepsilon}^{(q)}=\left(\mathbf{1}+Z^{\varepsilon}\right)\left(\mathbf{u}_{0}^{(q)}+\widehat{\mathbf{u}}_{\varepsilon}^{(q)}\right), \quad \widetilde{\mathbf{w}}_{\varepsilon}^{(q)}=\left(\mathbf{1}+Y^{\varepsilon}\right)\left(\mathbf{w}_{0}^{(q)}+\widehat{\mathbf{w}}_{\varepsilon}^{(q)}\right), \\
\widetilde{\mathbf{v}}_{\varepsilon}^{(q)}=\mathbf{v}_{0}^{(q)}+\widehat{\mathbf{v}}_{\varepsilon}^{(q)}, \quad \widetilde{\mathbf{z}}_{\varepsilon}^{(q)}=\mathbf{z}_{0}^{(q)}+\widehat{\mathbf{z}}_{\varepsilon}^{(q)} .
\end{gathered}
$$

Постоянные в оценках (2.45)-(2.48) зависят лишь от $\left|\mu_{0}\right|,\left|\mu_{0}^{-1}\right|,\|\eta\|_{L_{\infty}}$, $\left\|\eta^{-1}\right\|_{L_{\infty}}$ и от параметров решетки $\Gamma$.

Отметим, что поля $\mathbf{u}_{\varepsilon}^{(q)}, \mathbf{w}_{\varepsilon}^{(q)}, \mathbf{v}_{\varepsilon}^{(q)}, \mathbf{z}_{\varepsilon}^{(q)}$ слабо сходятся в $\mathfrak{G}$ к соответствующим усредненным полям. Поправки к усредненным полям, входящие в (2.49), (2.50), слабо сходятся к нулю, и их можно интерпретировать как корректоры нулевого порядка.

2.10. Частные случаи при $\mathbf{r}=\mathbf{0}$. Если $\eta^{0}=\underline{\eta}$, то $\widetilde{\eta}(\mathbf{x})=\eta^{0}$ и $Y(\mathbf{x})=0$, $\mathbf{1}+Z(\mathbf{x})=\eta(\mathbf{x})^{-1} \eta^{0}$. Тогда из (2.45), (2.46), (2.49) вытекает

Теорема 2.5. Пусть выполнены условия теоремы 2.4, причем $\eta^{0}=\underline{\eta}$. Тогда для полей $\mathbf{u}_{\varepsilon}^{(q)}, \mathbf{w}_{\varepsilon}^{(q)}$ справедливы оценки (2.45), (2.46), причем выражсения для $\widetilde{\mathbf{w}}_{\varepsilon}^{(q)}, \widetilde{\mathbf{u}}_{\varepsilon}^{(q)}$ принимают вид

$$
\widetilde{\mathbf{w}}_{\varepsilon}^{(q)}=\mathbf{w}_{0}^{(q)}+\widehat{\mathbf{w}}_{\varepsilon}^{(q)}, \quad \widetilde{\mathbf{u}}_{\varepsilon}^{(q)}=\left(\eta^{\varepsilon}\right)^{-1} \eta^{0}\left(\mathbf{u}_{0}^{(q)}+\widehat{\mathbf{u}}_{\varepsilon}^{(q)}\right)=\left(\eta^{\varepsilon}\right)^{-1}\left(\mathbf{w}_{0}^{(q)}+\widehat{\mathbf{w}}_{\varepsilon}^{(q)}\right) .
$$

Если $\eta^{0}=\bar{\eta}$, то $\Phi_{j}(\mathbf{x})=0, j=1,2,3$, а тогда и $Z(\mathbf{x})=0$. В этом случае решение поправочной системы тривиально. Далее, в данном случае $\widetilde{\eta}(\mathbf{x})=\eta(\mathbf{x})$, 
а потому $\mathbf{1}+Y(\mathbf{x})=\eta(\mathbf{x})\left(\eta^{0}\right)^{-1}$. Следовательно (см. (2.49), (2.50)),

$$
\widetilde{\mathbf{u}}_{\varepsilon}^{(q)}=\mathbf{u}_{0}^{(q)}, \quad \widetilde{\mathbf{w}}_{\varepsilon}^{(q)}=\eta^{\varepsilon}\left(\eta^{0}\right)^{-1} \mathbf{w}_{0}^{(q)}=\eta^{\varepsilon} \mathbf{u}_{0}^{(q)}, \quad \widetilde{\mathbf{v}}_{\varepsilon}^{(q)}=\mathbf{v}_{0}^{(q)}, \quad \widetilde{\mathbf{z}}_{\varepsilon}^{(q)}=\mathbf{z}_{0}^{(q)} .
$$

С учетом сказанного из теоремы 2.4 вытекает

Теорема 2.6. Пусть выполнены условия теоремы 2.4, причем $\eta^{0}=\bar{\eta}$. Тогда для полей $\mathbf{u}_{\varepsilon}^{(q)}, \mathbf{w}_{\varepsilon}^{(q)}, \mathbf{v}_{\varepsilon}^{(q)}, \mathbf{z}_{\varepsilon}^{(q)}$ справедливы следующие аппроксимащии по норме в $\mathfrak{G}$ :

$$
\begin{array}{cl}
\left\|\mathbf{u}_{\varepsilon}^{(q)}-\mathbf{u}_{0}^{(q)}\right\|_{\mathfrak{G}} \leqslant C_{6} \varepsilon\|\mathbf{q}\|_{\mathfrak{G},}, & 0<\varepsilon \leqslant 1, \\
\left\|\mathbf{w}_{\varepsilon}^{(q)}-\eta^{\varepsilon} \mathbf{u}_{0}^{(q)}\right\|_{\mathfrak{G}} \leqslant C_{7} \varepsilon\|\mathbf{q}\|_{\mathfrak{G},}, & 0<\varepsilon \leqslant 1, \\
\left\|\mathbf{v}_{\varepsilon}^{(q)}-\mathbf{v}_{0}^{(q)}\right\|_{\mathfrak{G}} \leqslant C_{5}^{\prime}\left|\mu_{0}^{-1}\left\|\left.\mu_{0}\right|^{1 / 2} \varepsilon\right\| \mathbf{q} \|_{\mathfrak{G},},\right. & 0<\varepsilon \leqslant 1, \\
\left\|\mathbf{z}_{\varepsilon}^{(q)}-\mathbf{z}_{0}^{(q)}\right\|_{\mathfrak{G}} \leqslant C_{5}^{\prime}\left|\mu_{0}\right|^{1 / 2} \varepsilon\|\mathbf{q}\|_{\mathfrak{G}}, & 0<\varepsilon \leqslant 1 .
\end{array}
$$

Постоянные в оценках (2.51)-(2.54) зависят лишь от $\left|\mu_{0}\right|,\left|\mu_{0}^{-1}\right|,\|\eta\|_{L_{\infty}}$, $\left\|\eta^{-1}\right\|_{L_{\infty}}$ и от параметров решетки $Г$.

2.11. Сводный результат. Теперь мы объединим результаты для случаев $\mathbf{q}=0$ и $\mathbf{r}=0$, несколько огрубив их. Из п. $1^{\circ}$ теоремы 2.1 и теоремы 2.4 вытекает

Теорема 2.7. Пусть $\left(\mathbf{w}_{\varepsilon}, \mathbf{z}_{\varepsilon}\right)$ - решение системъ $(2.3)$ u $\mathbf{u}_{\varepsilon}=\left(\eta^{\varepsilon}\right)^{-1} \mathbf{w}_{\varepsilon}$, $\mathbf{v}_{\varepsilon}=\mu_{0}^{-1} \mathbf{z}_{\varepsilon}$. Пусть $\left(\mathbf{w}_{0}, \mathbf{z}_{0}\right)$ - решение «усредненной» системь (2.4) $u \mathbf{u}_{0}$, $\mathbf{v}_{0}$ определены в (2.5). Пусть $\left(\widehat{\mathbf{w}}_{\varepsilon}^{(q)}, \widehat{\mathbf{z}}_{\varepsilon}^{(q)}\right)$ - решение «поправочной» системы (2.40) и $\widehat{\mathbf{u}}_{\varepsilon}^{(q)}, \widehat{\mathbf{v}}_{\varepsilon}^{(q)}$ определенъ в (2.41). Пусть матрицъ $Z(\mathbf{x}), Y(\mathbf{x})$ - те же , что и в условиях теоремы 2.1. Тогда для всех четырех полей $\mathbf{u}_{\varepsilon}, \mathbf{w}_{\varepsilon}, \mathbf{v}_{\varepsilon}, \mathbf{z}_{\varepsilon}$ справедливы аппроксимачии по норме в $\mathfrak{G}$ с погрешностью порядка є:

$$
\begin{array}{ll}
\left\|\mathbf{u}_{\varepsilon}-\left(\mathbf{1}+Z^{\varepsilon}\right)\left(\mathbf{u}_{0}+\widehat{\mathbf{u}}_{\varepsilon}^{(q)}\right)\right\|_{\mathfrak{G}} \leqslant \varepsilon\left(C_{5}^{\prime \prime}\|\mathbf{r}\|_{\mathfrak{G}}+C_{6}\|\mathbf{q}\|_{\mathfrak{G}}\right), & 0<\varepsilon \leqslant 1, \\
\left\|\mathbf{w}_{\varepsilon}-\left(\mathbf{1}+Y^{\varepsilon}\right)\left(\mathbf{w}_{0}+\widehat{\mathbf{w}}_{\varepsilon}^{(q)}\right)\right\|_{\mathfrak{G}} \leqslant \varepsilon\left(C_{5}^{\prime \prime}\|\eta\|_{L_{\infty}}\|\mathbf{r}\|_{\mathfrak{G}}+C_{7}\|\mathbf{q}\|_{\mathfrak{G}}\right), & 0<\varepsilon \leqslant 1, \\
\left\|\mathbf{v}_{\varepsilon}-\left(\mathbf{v}_{0}+\widehat{\mathbf{v}}_{\varepsilon}^{(q)}\right)\right\|_{\mathfrak{G}} \leqslant \varepsilon\left|\mu_{0}^{-1}\right|\left(C_{1}^{\prime \prime}\|\mathbf{r}\|_{\mathfrak{G}}+C_{5}^{\prime}\left|\mu_{0}\right|^{1 / 2}\|\mathbf{q}\|_{\mathfrak{G}}\right), & 0<\varepsilon \leqslant 1, \\
\left\|\mathbf{z}_{\varepsilon}-\left(\mathbf{z}_{0}+\widehat{\mathbf{z}}_{\varepsilon}^{(q)}\right)\right\|_{\mathfrak{G}} \leqslant \varepsilon\left(C_{1}^{\prime \prime}\|\mathbf{r}\|_{\mathfrak{G}}+C_{5}^{\prime}\left|\mu_{0}\right|^{1 / 2}\|\mathbf{q}\|_{\mathfrak{G}}\right), & 0<\varepsilon \leqslant 1 .
\end{array}
$$

Обсудим частные случаи. Справедлива

Теорема 2.8. Пусть выполнены условия теоремы 2.7, причем $\eta^{0}=\underline{\eta}$. Тогда оценки (2.55) и (2.56) принимают вид

$$
\begin{array}{ll}
\left\|\mathbf{u}_{\varepsilon}-\left(\eta^{\varepsilon}\right)^{-1}\left(\mathbf{w}_{0}+\widehat{\mathbf{w}}_{\varepsilon}^{(q)}\right)\right\|_{\mathfrak{G}} \leqslant \varepsilon\left(C_{5}^{\prime \prime}\|\mathbf{r}\|_{\mathfrak{G}}+C_{6}\|\mathbf{q}\|_{\mathfrak{G}}\right), & 0<\varepsilon \leqslant 1, \\
\left\|\mathbf{w}_{\varepsilon}-\left(\mathbf{w}_{0}+\widehat{\mathbf{w}}_{\varepsilon}^{(q)}\right)\right\|_{\mathfrak{G}} \leqslant \varepsilon\left(C_{5}^{\prime \prime}\|\eta\|_{L_{\infty}}\|\mathbf{r}\|_{\mathfrak{G}}+C_{7}\|\mathbf{q}\|_{\mathfrak{G}}\right), & 0<\varepsilon \leqslant 1 .
\end{array}
$$

Объединяя результаты теоремы 2.3 и теоремы 2.6 (см. (2.51), (2.52)), приходим к следующему утверждению.

Теорема 2.9. Пусть выполнены условия теоремы 2.7, причем $\eta^{0}=\bar{\eta}$. Тогда

$$
\begin{aligned}
\left\|\mathbf{u}_{\varepsilon}-\mathbf{u}_{0}\right\|_{\mathfrak{G}} & \leqslant \varepsilon\left(C_{5}^{\prime \prime}\|\mathbf{r}\|_{\mathfrak{G}}+C_{6}\|\mathbf{q}\|_{\mathfrak{G}}\right), & & 0<\varepsilon \leqslant 1, \\
\left\|\mathbf{w}_{\varepsilon}-\eta^{\varepsilon} \mathbf{u}_{0}\right\|_{\mathfrak{G}} & \leqslant \varepsilon\left(C_{5}^{\prime \prime}\|\eta\|_{L_{\infty}}\|\mathbf{r}\|_{\mathfrak{G}}+C_{7}\|\mathbf{q}\|_{\mathfrak{G}}\right), & & 0<\varepsilon \leqslant 1 .
\end{aligned}
$$




\section{ЛитЕРАТУРА}

[1] Н. С. Бахвалов, Г. П. Панасенко, Осреднение процессов в периодических средах, Наука, М., 1984.

[2] A. Bensoussan, J.-L. Lions, G. Papanicolau, Asymptotic analysis for periodic structures, North-Holland Publishing Co., Amsterdam-New York, 1978.

[3] M. Sh. Birman, T. A. Suslina, Threshold effects near the lower edge of the spectrum for periodic differential operators of mathematical physics, in: Oper. Theory: Adv. Appl., vol. 129, Birkhäuser, Basel, 2001, 71-107.

[4] М. Ш. Бирман, Т. А. Суслина, Периодические дифференциальные операторы второго порядка. Пороговые свойства и усреднения, Алгебра и анализ, 15:5 (2003), $1-108$.

[5] М. Ш. Бирман, Т. А. Суслина, Пороговые аппроксимации резолъвенты факторизованного самосопряженного семейства с учетом корректора, Алгебра и анализ, 17:5 (2005), 69-90.

[6] М. Ш. Бирман, Т. А. Суслина, Усреднение периодических эллиптических дифференииальных операторов с учетом корректора, Алгебра и анализ, 17:6 (2005), $1-104$.

[7] М. Ш. Бирман, Т. А. Суслина, Усреднение периодических дифференциальных операторов с учетом корректора. Приближение решений в классе Соболева $H^{1}\left(\mathbb{R}^{d}\right)$, Алгебра и анализ, 18:6 (2006), 1-130.

[8] В. В. Жиков, С. М. Козлов, О. А. Олейник, Усреднение дифференциалъных операторов, Физматгиз, М., 1993.

[9] Э. Санчес-Паленсия, Неоднородные средъ и теория колебаний, Мир, М., 1984.

[10] Т. А. Суслина, Об усреднении периодической системы Максвелла, Функц. анализ и его прил., 38:3 (2004), 90-94.

[11] Т. А. Суслина, Усреднение стационарной периодической системь Максвелла, Алгебра и анализ, 16:5 (2004), 162-244.

Санкт-Петербургский государственный университет, физический факультет

Поступило в редакцию email: mbirman@list.ru 30 ноября 2006 г.

Санкт-Петербургский государственный университет, физический факультет email: suslina@list.ru 